This is the penultimate version of this paper. The final version is published in Studies in History and Philosophy of Modern Physics: http://

wWw.sciencedirect.com/science/article/pii/s1355219816300739

DOI information: 10.1016/j.shpsb.2016.08.003

\title{
The Higgs mechanism and superconductivity: A case study of formal analogies
}

\author{
Doreen Fraser*1 and Adam Koberinski ${ }^{\dagger 2}$ \\ ${ }^{1}$ University of Waterloo, Department of Philosophy , 300 University Ave. W. , Waterloo, \\ ON N2L 3G1 Canada \\ ${ }^{2}$ Western University, Rotman Institute, 1151 Richmond St. , London, ON N6A 5B8 \\ Canada
}

\begin{abstract}
Following the experimental discovery of the Higgs boson, physicists explained the discovery to the public by appealing to analogies with condensed matter physics. The historical root of these analogies is the analogies to models of superconductivity that inspired the introduction of spontaneous symmetry breaking (SSB) into particle physics in the early 1960s. We offer a historical and philosophical analysis of the analogies between the Higgs model of the electroweak (EW) interaction and the Ginsburg-Landau (GL) and Bardeen-Cooper-Schrieffer (BCS) models of superconductivity, respectively. The conclusion of our analysis is that both sets of analogies are purely formal in virtue of the fact that they are accompanied by substantial physical disanalogies. In particular, the formal analogies do not map the temporal, causal, or modal structures of SSB in superconductivity to temporal, causal, or modal structures in the Higgs model. These substantial physical disanalogies mean that analogies to models of superconductivity cannot supply the basis for the physical interpretation of EW SSB; however, an appreciation of the contrast between the physical interpretations of SSB in superconductivity and the Higgs model does help to some foundational issues. Unlike SSB in superconductivity, SSB in the Higgs sector of the Standard Model (without the addition of new physics) is neither a temporal nor a causal process. We discuss the implications for the 'eating' metaphor for mass gain in the Higgs model. Furthermore, the distinction between the phenomenological GL model and the dynamical BCS model does not carry over to EW models, which clarifies the desiderata for so-called 'dynamical' models of EW SSB (e.g., minimal technicolor). Finally, the development of the Higgs model is an illuminating case study for philosophers of science because it illustrates how purely formal analogies can play a fruitful heuristic role in physics.
\end{abstract}

\section{Introduction}

The Higgs mechanism, and the application of spontaneous symmetry breaking (SSB) in particle physics more widely, has historical origins in condensed matter physics. According to

*dlfraser@uwaterloo.ca; Corresponding author

†akoberin@uwo.ca 
the Bardeen-Cooper-Schrieffer (BCS) model of superconductivity, low-temperature superconductivity occurs when the ground state of a metal has a lower symmetry than the solid itself (at sufficiently low temperatures), and when the metal is in this state, electrons condense together to form bound states (Cooper pairs). Nambu noticed (in 1960) the formal similarities between the BCS model and the Dirac equation, and so borrowed the concept for what turned out to be an effective theory of quantum chromodynamics (QCD). With this move, SSB became a valuable heuristic tool in particle physics. It was employed by Higgs, Englert, Brout, and others in 1964 to introduce massive gauge bosons into quantum field theory. The Higgs mechanism ended up being a key ingredient in the renormalizable theory produced by the Glashow-Weinberg-Salam (GWS) electroweak (EW) unification (1967).

The aim of this paper is to determine what analogies to superconductivity reveal about the physical interpretation of the Higgs mechanism. While there is a tradition of using analogies to condensed matter physics to explain the Higgs mechanism and the Higgs boson to a lay audience and to physics students, there has been a recent resurgence in such explanations with the celebrated discovery of the Higgs boson at the Large Hadron Collider. One of the best analogies of this type is David Miller's from 1993, for which he won a bottle of champagne in a competition from then U.K. Minister of Science William Waldegrave (and which played a role in the UK's approval of funding for the Large Hadron Collider). Miller draws his analogy between three domains: British politics, solid state physics, and the Higgs model. A room full of politicians evenly distributed is taken as the analogue of the Higgs vacuum. As a noteworthy politician enters the room, the distribution of people distorts to cluster around her, providing resistance to her motion through the room. The politician is the analogue of a $\mathrm{W}$ or $\mathrm{Z}$ boson, the people clustering around her represent the interaction between a particle and the Higgs field, and the resisted motion is the analogue of the acquisition of mass. Likewise, a rumor of scandal can cause clustering in the room, and this cluster travels as the rumor spreads amongst the politicians. The cluster occurs without an external politician (particle) present, and these clusters in the politician 'field' are the analogues of the Higgs boson (Miller, 1993). Analogies such as this help the public and physics students gain intuitive understanding of the formal framework of the Higgs mechanism. Whether the analogies also convey information about the physical interpretation of the formalism depends on a more detailed analysis of the types of analogies that have been successfully employed. Our main analytical tool will be to clearly distinguish formal analogies between superconductivity and EW SSB from material and physical analogies. We argue that the Higgs model demonstrates that purely formal analogies can play a fruitful heuristic role in physics; this is a case in which formal analogies are not underwritten by a further physical or material analogy, yet have proven to be very useful.

The Higgs mechanism is a remarkably good case study of the heuristic use of analogies in contemporary physics because it is widely recognized that there are two models of superconductivity - the BCS and Ginzburg-Landau (GL) models - which support different types of analogies. The BCS model is a dynamical model that offers a dynamical mechanism for the transition from 'normal' to superconducting states (i.e., the formation of Cooper pairs). In contrast, the GL model, first proposed by Ginzburg and Landau prior to the 
BCS model, is (merely) a phenomenological model because it does not offer a dynamical mechanism for superconductivity. Formally, the Higgs model is clearly a closer analogue to the GL model than the BCS model. The analogies to the two models of superconductivity are widely recognized in historical accounts of the discoveries. For example, in his Nobel prize address, Higgs explains that "Nambu's models were inspired by the Bardeen, Cooper, and Schrieffer theory (Bardeen et al., 1957), based on Bose condensation of Cooper pairs of electrons: Goldstone used scalar fields, with a "wine bottle" potential to induce Bose condensation, as in the earlier Ginzburg-Landau theory" (2014, p. 851). ${ }^{1}$ There are also good treatments of analogies to both models in the contemporary physics textbook and review article literature. (For example, Marshak (1993), Quigg (2007), and Witten (2007) offer clear presentations.) The success ${ }^{2}$ of the analogies to superconductivity in providing a heuristic for formulating the Higgs model set another program in motion: the program of devising a new model for electroweak interactions that is more closely analogous to the BCS model than the GL model. A number of such dynamical SSB models have been proposed, including the quark composite model, the electroweak perturbation model, and different versions of technicolor.

The first part of the paper is devoted to a historical and philosophical analysis of the types of analogies that hold between the Higgs model and the GL and BCS models, respectively. For this purpose, we distinguish between three types of analogies: formal, physical and material. We use Hesse's account of analogies to frame our discussion. Roughly, formal analogies map similar elements of the mathematical formalisms of the models; physical analogies map elements of the models with similar physical interpretations; and material analogies map the causal structures of the models. In Sec. 3 we give brief expositions of the GL model, the BCS model, and the Higgs model. After identifying the formal analogies between the BCS and GL models, respectively, and the Higgs model in Sec. 4, we turn to the prospects for physical and material analogies between the models in Sec. 5. The formal analogical mappings do not map the temporal, modal, or causal structures of the superconductivity models to temporal, modal, or causal structures in the Higgs model. We argue that these crucial differences between the physical interpretations of the analogues rule out material and physical analogies between the BCS and GL models, respectively, and the Higgs model. Furthermore, the distinction between phenomenological and dynamical models gleaned from the GL and BCS models is not applicable to the Higgs model. The material and physical disanalogies between the superconductivity models and the Higgs model also entail that neither the BCS model nor the GL model supplies a guide for the physical interpretation of the Higgs field or the Higgs boson.

\footnotetext{
${ }^{1}$ In the historical literature, Brown and Cao opine that "Nambu's approach is 'microscopic', and comparable to the BCS-Bogoliubov theory" whereas "Goldstone's is 'macroscopic', in the sense of 'phenomenological', comparable to the Landau-Ginzburg theory" (Brown and Cao, 1991, p. 232).

In his Nobel prize lecture, Nambu notes that "[t]he [Weinberg-Salam theory of electroweak unification] resembles the Ginzburg-Landau description of superconductivity, which was shown to follow from the BCS theory by Gor'kov" (Nambu, 2008, p.62).

${ }^{2}$ At least conceptually; the direct empirical success of the Higgs model of course took much longer to establish.
} 
Our treatment of the Higgs mechanism is for the most part orthogonal to one of the dominant strands in the philosophical literature. Earman (2002) draws attention to the problem that, according to standard presentations of the Higgs mechanism in the contemporary physics literature, particles gain mass as a result of choosing a particular gauge; this is at odds with philosophical accounts according to which a gauge symmetry is supposed to represent "descriptive fluff," a mere redundancy in the mathematical representation without physical content. Earman thus demands that an adequate account of the Higgs mechanism be gauge invariant in order to determine its physical interpretation. Our discussion of the Higgs mechanism is for most part orthogonal to these issues because we focus on historical presentations of the Higgs model. Analogies to superconductivity were most influential in the development of the EW component of the Standard Model c.1960-6. At this time gauge invariant presentations of SSB were current (Struyve (2011)). As a result, the analogies examined here do not hinge on the choice of a particular gauge. The mass gain metaphor will be discussed in its historical context in Sec. 5.5.

While our main conclusion is the negative one that models of superconductivity do not help to inform the physical interpretation of the Higgs mechanism, the points of contrast with superconductivity do serve to clarify some foundational issues, particularly regarding the role of time in the Higgs model. We emphasize that the scope of our arguments in Sections 3-5 (i.e., excluding Sec. 6) is limited to the Higgs mechanism as presented in the Standard Model of particle physics. We take it that this focus on the theoretical presentation of EW SSB in the Standard Model is in keeping with most of the philosophical discussion of EW SSB to date. To clarify, questions about the physical interpretation of SSB in the Higgs sector of the Standard Model are questions about the possible world described by the Higgs model. This possible world may well not be the actual world. In particular, one of the main points that we want to stress to our philosophical audience is that - unlike SSB in superconductivitySSB in the Higgs model is not a temporal process. This is a partial answer to questions raised by Huggett in (Cao, 1999, pp.376-377) and Cao in (Hoddeson, 1997, pp. 504-505). Of course, it may turn out that in our actual world EW SSB is a temporal process (e.g., that a phase transition occurred in the early universe). This type of description could only be offered by a model of EW SSB other than the Higgs model. Indeed, it is difficult to imagine how physicists could reach the conclusion that an EW phase transition occurred in the early universe without having developed a model that describes such a transition. Finite temperature field theory (aka thermal field theory) was devised in part to provide a theoretical framework for SSB in cosmology. The aim of finite temperature field theory is to present a single theoretical framework that both contains a temperature variable and is a (relativistic) quantum field theory (see, e.g., Le Bellac (2000)). Finite temperature field theory presents new physics that goes beyond the Standard Model, and its project of amalgamating statistical mechanics and QFT goes well beyond the strict analogies between QFT and condensed matter systems considered here. Philosophical discussions of EW SSB sometimes lump the Higgs model and finite temperature field theory together and describe the EW SSB as a phase transition that possibly occurred once in the early universe (e.g., (Wuthrich, 2012, p.10, footnote 6)). We hope that this paper will serve as encouragement 
for philosophers interested in EW SSB as a temporal process to attend to theories that go beyond the Standard Model.

In Sec. 6, we waive the restriction in force in the rest of the paper in order to consider current and past attempts to go beyond the Standard Model (BSM models) by constructing a model for EW SSB that is more closely analogous to the BCS model (e.g., the minimal technicolor model). The strategy common to these so-called dynamical models is to introduce a composite particle that is analogous to the Cooper pair. We argue that, since the Higgs model cannot be considered a phenomenological model in the same sense as the GL model, these 'dynamical' models cannot be regarded as supplying a causal-dynamical underpinning for the Higgs model in the manner that the BCS model supplies a causal-dynamical underpinning for the GL model. However, there remain other motivations for pursuing these 'dynamical' SSB models. Pushing analogies with superconductivity as far as possible was (and is) a reasonable heuristic to employ for developing new Lagrangian models of the EW interaction. As is the case for other applications of analogical reasoning in the history of physics, the measure of success for the resulting models is passing empirical tests. Two of the dynamical SSB models discussed below have failed empirical tests and the verdict is still out on the third (minimal technicolor). (By comparison, so far the Higgs model has passed empirical tests.)

In this paper we focus on the aspects of the history that are relevant to philosophical and interpretive issues under consideration. As a result, our presentation of the history is not comprehensive. For further historical background, consult Hoddeson (1997) and recent papers by the Wuppertal research collaboration on the epistemology of the Large Hadron Collider (e.g., Stöltzner (2014)). We regard our analysis of analogies with superconductivity as complementary to the thesis in Koraca (2011) that there is "a practical unity" between superconductivity and the Higgs mechanism which, historically, was a product of the methods for SSB in both being co-developed between the fields of condensed matter and high energy physics. Another historical case study of the utility of formal analogies in physics is to be found in Gingras (2015), which studies Einstein's discovery of wave-particle duality.

\section{Material, physical, and formal analogies}

The general accounts of analogies in science offered by Hesse, Bartha (articulation model), and Holyoak and Thagard (multi-constraint theory) disagree on many details, but are in agreement that analogies have played an important heuristic role in many scientific discoveries. They also agree that analogical reasoning does not take the form of deductive argument; empirical testing is what determines whether an analogical inference has a true conclusion. The applications of analogies to the BCS model in particle physics fit into this general template: they served as a useful heuristic for producing analogical models of EW SSB which have been (and continue to be) subject to empirical test.

For our purposes, it is important to distinguish between different types of analogies. We will draw on distinctions between material, physical, and formal analogies. To frame these distinctions, consider Hesse's account. Hesse introduced the now-familiar distinction between 
horizontal and vertical relations: horizontal relations are mappings between objects in the source and target domains and vertical relations are mappings between objects within one domain. For example, in an analogy drawn between the earth (source domain) and the moon (target domain), horizontal relations could include that the bodies are both approximately spherical and that the earth has an atmosphere while the moon has no atmosphere (1966, pp. 58-59). The similarity in respect of being approximately spherical is a positive analogy and the difference in respect of having an atmosphere is a negative analogy. The presence of human beings could be considered a neutral analogy: it is not known (in 1966) whether human beings are present on the moon. An argument from analogy may be formulated with the positive and negative analogies (i.e., horizontal relations) between the earth and moon and the presence of humans on earth as premises and the presence of humans on the moon as the conclusion. Hesse contends that the strength of arguments from analogy rests on the causal relations between the properties that are related through positive and negative analogies. Causal relations are Hesse's paradigm case of vertical relations. ${ }^{3}$ For example, the presence of an atmosphere on earth is known to be causally related to sustaining human life on earth; this is a vertical relation within the source domain. The fact that presence of an atmosphere is part of the negative analogy undermines the argument from analogy to the conclusion that there are humans on the moon.

Hesse introduced a distinction between material and formal analogies. Material analogies invoke "pre-theoretic analogies between observables ${ }^{4} \ldots$ which enable predictions to be made from a model" (p. 68). A further condition on material analogies is that the vertical relations invoked be causal relations of the same kind in both the source and target domains. In contrast, a formal analogy involves only "the one-to-one correspondence between different interpretations of the same formal [i.e., uninterpreted] theory" (p. 68). Put another way, formal analogies are cases in which there is "no horizontal similarity independent of the vertical relation" (p. 68). The paradigm case of a formal analogy is the application of the same equation (with different physical interpretations) in distinct domains. An analogy is merely formal - and not material - if the two physical theories employing this equation give an interpretation that does not invoke causal relations of the same kind.

There is another type of analogy that is also relevant. Horizontal relations can reflect physical similarities between the source and target domains without being implicated in causal vertical relations. Such horizontal relations are not material, but not purely formal either. We shall label this intermediate case physical analogy. Bartha (2010) also distinguishes this category of analogy (pp. 207-210).

To sum up, these three types of analogy are related as follows: material analogies are also physical and formal; physical analogies may also be formal (depending on the vertical

\footnotetext{
${ }^{3}$ Causal relations are also of central importance for Holyoak and Thagard (1995), though for different reasons: causal relations are the paradigm case of higher-order structural relations and analogies which map higher-order relations are stronger because this increases the degree of mapping between the structures of the source and target systems (pp. 36-37).

${ }^{4}$ Though Hesse focuses on observables, we generalize our focus to theoretical variables. Entities or variables in the models that aren't observable can contribute to material analogies if they are to be interpreted as physically significant by the lights of the theory.
} 
relations), but not material; formal analogies may be neither material nor formal. Formal analogies are compatible with material and physical disanalogies that block the transfer of the physical interpretation from one model to the other.

Since the initial work by Nambu and Jona-Lasinio (among others) in the early 1960s, physicists have developed models for electroweak SSB based on different types of analogies to superconductivity. The different types of analogies correspond to the use of different models of SSB in superconductivity as analogues. As we shall see, the BCS model is a causal-dynamical model and the GL model is a phenomenological model which does not posit a causal-dynamical mechanism for the breaking of the symmetry. Consequently, only the BCS model can support material analogies. Analogies to the GL model are formal; we will investigate whether they are also physical. After providing an overview of the GL, BCS, and Higgs models, our approach will be to first identify the formal analogies between the GL and the Higgs models and the BCS and the Higgs models, and then to determine whether these analogies are also physical (or material, in the case of the BCS model). Our conclusion is that this is a case in which the formal analogies are accompanied by physical disanalogies rather than analogies. In Sec. 6 we will consider the status of the analogies to the BCS model invoked by several of the dynamical SSB models that have been proposed as alternatives to the Higgs model.

\section{The GL, BCS, and Higgs models}

Anderson and Nambu were among the first physicists to recognize analogies between the BCS model of superconductivity and particle physics. The features of the model that attracted their attention were the presence of a 'heavy' photon (i.e., a photon with effective mass) and the absence of massless Goldstone bosons. In Nambu's words, in a superconductor the Goldstone "mode mixes with the coulomb [sic] interaction among the electrons because of their common long-range nature, and turns into the well-known plasmons" (Nambu, 2008, p. 60). From the perspective of particle physics, both features were noteworthy because they contradicted widely held beliefs in the particle physics community. Circa 1962 particle physicists believed that the gauge bosons in Yang-Mills type theories - such as the photon in electromagnetism - are necessarily massless. Furthermore, on the strength of Goldstone's theorem, particle physicists believed that broken symmetries are necessarily accompanied by massless Goldstone bosons, and experiments had not turned up unaccounted-for massless particles. ${ }^{5}$ Goldstone's theorem - that continuous symmetries that are spontaneously broken in relativistic QFT are necessarily accompanied by massless zero-spin particles ("Goldstone bosons") was conjectured in Goldstone (1961) and proven in Goldstone et al. (1962).

Nambu first used the analogy from the BCS model of superconductivity to formulate a particle physics model. The idea of carrying the symmetry breaking apparatus over to a particle physics setting was motivated simply by the formal similarity of the Bogoliubov-

\footnotetext{
${ }^{5}$ Jona-Lasinio (2003) points out a third misconception of particle physicists in the late 1950s: the "strict dogma" that the vacuum "should not possess observable physical properties and all the symmetries of the theory, implemented by unitary operators, should leave it invariant" (p. 316).
} 
Valantin equations ((6) below) to the Dirac equation. The mapping is made explicit by Nambu:

the gap $\Delta$ goes over to the mass $M$, which breaks chirality $\sim \gamma_{5}$ rather than the ordinary charge $\sim 1$ : the axial current is the analog of the electromagnetic vector current in the BCS theory... [C]hiral symmetry is compatible with a finite nucleon mass $M$ provided that there exists a massless pseudoscalar [Goldstone] boson. (p. 60)

This work was continued further by Nambu and Jona-Lasinio (NJL), who constructed an effective model of composite nucleons based on the analogy with BCS theory, in which nucleons played the role of composite Cooper pairs, and the energy gap of the BCS theory was replaced by the resulting mass of the nucleons. The analogous broken symmetry in this model is the global $U(1)$ chiral symmetry. Regarding the pions as approximately massless (compared to the larger nucleons), they play the role of the Goldstone bosons upon SSB. As Nambu explains, from a contemporary perspective, the NJL model "may be regarded as an effective theory for QCD with respect to generation of the so-called constituent masses. One is interested in the low energy degrees of freedom on a scale smaller than some cut-off $\Lambda "$ (2008, p. 62).

The NJL model hinted at the possibility of solving the problem of massive mediating bosons in Yang-Mills gauge theories through SSB. However, Goldstone's theorem posed an obstacle to this task because the massless Goldstone bosons accompanying SSB should have been easy to produce in experiments. Ironically, examples of Goldstone bosons in solid state physics (e.g., phonons, spin waves) may have served as inspiration for Goldstone's theorem (Anderson, 1963, p. 441). As Anderson recalled many years later,

Another roadblock [to EW theory] was the apparent necessity of allowing a number of Goldstone bosons into the theory, which would mean that the theory would be full of massless bosons - which didn't exist! When I heard in 1962 that people considered this a real obstacle, I sent off a short paper saying "forget it - the gap is empty in a real superconductor!" (2015).

Roughly speaking, the intuitive lesson drawn from superconductivity was that there are in fact no zero mass problems in theories in which gauge bosons and Goldstone bosons are suitably coupled. To put the point in an uncontroversial but less colorful way, models of superconductivity demonstrated that particle physicists' separate assumptions about the necessity of massless gauge bosons and massless Goldstone bosons failed when combined (i.e., in the context of gauge theories with spontaneously broken symmetries). In Higgs' words,

...the spontaneous breakdown program of Nambu et al and the gauge field program of Salam et al stand or fall together. Each saves the other from the zeromass difficulty. (1966, p. 145) 
Anderson has a more colorful way of stating the moral: "We conclude, then, that the Goldstone zero-mass difficulty is not a serious one, because we can probably cancel it off against an equal Yang-Mills zero-mass problem" (1963, p. 442). As these quotes indicate, theories of superconductivity and proposed quantum field theories were judged relevantly similar in virtue of being Yang-Mills-type theories; this was the primary meaning accorded to the term gauge theory (see Weatherall (2015)).

Higgs (1964), among others, took up Anderson's suggestion, and showed how SSB of a gauge symmetry could eliminate the Goldstone boson, resulting in a massive vector boson and a massive scalar boson, now known as the Higgs boson. The mechanism was applied by Weinberg (1967) - building on the work of Salam and Glashow - to the non-Abelian $S U(2) \times$ $U(1)$ symmetry group unifying electromagnetism and the weak force. The resulting theory is known as GWS electroweak theory, which is a staple of the Standard Model of particle physics. $^{6}$

While historically the BCS model was the superconductivity analogue that played the central role in this sequence of developments, its precursor - the GL model - is important for the analysis of the analogies. The dynamical BCS model entails the phenomenological GL model in the sense that (given suitable background assumptions) near the critical temperature the GL equations can be derived from the BCS equations. The remainder of this section outlines all three of the models - GL, BCS, and Higgs.

\subsection{The Ginzburg-Landau phenomenological model for supercon- ductivity}

The Ginzburg-Landau (GL) model was an attempt from 1950 to build a phenomenological model of superconductivity. For this reason, Ginzburg and Landau did not attempt to justify certain choices made within their model, beyond appeal to consistency and agreement with experiment. They note that, in any situation involving second order phase transitions, "there always enters some parameter [which] differs from zero in the ordered phase and which equals zero in the disordered phase" (Ginzburg and Landau, 2009, p. 115). Since the transition to a superconducting state is a second order phase transition, they propose an order parameter $\psi$, which can be interpreted as representing an effective wavefunction for the superconducting electrons, ${ }^{7}$ that vanishes above some critical temperature, $T_{c}$. Starting from the general expansion of free energy about the critical temperature - the lowest order expansion that accommodates the phenomena - in the absence of other interactions, the free energy density

\footnotetext{
${ }^{6}$ This historical overview is meant to provide a streamlined narrative of the events leading from BCS theory to the Higgs mechanism in electroweak theory. For a more thorough account, see Koraca (2011), or Hoddeson (1997).

${ }^{7}$ This is the contemporary interpretation. As Kadanoff (2013) persuasively argues, the interpretation of $\psi$ as representing a collection of particles - the superconducting electrons - was a moral that took years for both the Russian and American physics communities to fully absorb. Ginzburg and Landau originally proposed a microscopic definition of $\psi$ (Kadanoff, 2013, pp. 814-5). Kadanoff presents evidence that as late as 1962 Bardeen himself did not recognize the significance of the collective wavefunction (pp. 817-8).
} 
can be written as

$$
\mathcal{F}_{s}=\mathcal{F}_{n}+a|\psi|^{2}+\frac{b}{2}|\psi|^{4}
$$

where $\mathcal{F}_{n}$ represents the free energy density of the 'normal' metal (i.e., the metal in the 'normal', as opposed to superconducting, state). This general form was previously confirmed for other systems undergoing second order phase transitions, and was thus considered a reasonable hypothesis. (Presumably this is another formal analogy!) In addition to these terms they include the energy density given by a constant magnetic field, $H^{2} / 2 \pi$, and the gauge covariant derivative linking wavefunction $\psi$ to the electromagnetic field, in analogy with the Schrödinger equation coupled to the electromagnetic field. The final form of the free energy density is given by

$$
\mathcal{F}_{s}=\mathcal{F}_{n}+\left|D_{i} \psi\right|^{2}+a|\psi|^{2}+\frac{b}{2}|\psi|^{4}+\frac{H^{2}}{8 \pi}
$$

with $D_{i}=\frac{1}{2 m^{*}}\left(\nabla-i e^{*} A\right)$ the three-dimensional covariant derivative and $m^{*}$ and $e^{*}$ empiricallydetermined parameters. By requiring that $|\psi|^{2}>0$ for $T<T_{c}$, a is forced to be negative. Along with certain other constraints on the free energy (e.g. order parameter continuously approaches 0 at $T \rightarrow T_{c}$ ), the simplest form for the order parameter (for $T \leq T_{c}$ ) is

$$
|\psi|^{2}=\frac{T_{c}-T}{b_{c}}\left(\frac{d a}{d T}\right)_{c},
$$

where $b_{c}$ is the value of the parameter $b$ at $T_{c}$, and both $a$ and $b$ are assumed to be functions of temperature. From these basic equations, many of the phenomenological features of superconductors are derived. ${ }^{8}$

The GL model is widely regarded as a phenomenological model, in contrast to the dynamical BCS model of superconductivity. The following features characterize the GL model as phenomenological:

1. descriptions: The GL model quantifies the phenomena related to superconductivity, including SSB. The order parameter goes from zero to a finite value when the temperature goes below $T_{c}$. This is a description of the process of SSB, but not an explanation of the underlying causes.

2. collections of constituents: $\psi(\mathbf{x})$ is the condensate wave function, which represents the collection of superconducting electrons (e.g., as opposed to one or two elementary particles). The GL model focuses on a collective description of the phenomena.

3. parameters are empirical inputs: Parameters $a, b, m^{*}$, and $e^{*}$ are not specified by the model. They are empirical inputs or free parameters which are set to make the model

\footnotetext{
${ }^{8}$ For a presentation geared toward mathematicians, see Witten (2007). This presentation was meant to retain the original heuristic flavour of the model as presented by Ginzburg and Landau in 1950.
} 
fit with the results of experiments. For example, the penetration depth $d$ and the coherence length $l$, both measurable quantities, are given by:

$$
d=\sqrt{\frac{m^{*} b}{4 \pi e^{*} a}} ; \quad l=\frac{1}{\sqrt{2 m^{*}|a|}},
$$

which provide constraints on the relationships between the free parameters. Only when the GL equations are derived from the BCS equations (including the micro-physical description of Cooper pairs) can it be determined that the parameters $e^{*}$ and $m^{*}$ are twice the electron's charge and mass, respectively.

\subsection{The BCS dynamical model of superconductivity}

At the time the BCS model was proposed, many phenomenological models of superconductivity already existed, including the GL model. One of the successes of the BCS model was that it provided a micro-level description of superconductivity, and served to provide a satisfying causal explanation of the phenomenon. In order to examine the details of the analogy between SSB in the Higgs and BCS models, it will be necessary to survey some of the details of the BCS model. This presentation follows Emch (2007, section 5.1), and is meant as a brief explication of the relevant aspects of the formalism. For more details, see Bardeen et al. (1957).

The starting points for the BCS model are Cooper pairs, thought to be "molecules" of two electrons interacting via a mediating phonon. Phonons are collective excitations associated with the quantized vibrational modes of the ionic lattice in the metal. This interaction was found to be attractive for electrons of anticorrelated spin and momentum. The Hamiltonian taken to represent the electron-phonon interaction in a large solid is

$$
H_{\Lambda}=\sum_{p, s} \omega_{p} a_{s, p}^{*} a_{s, p}+\sum_{p, p^{\prime}} b_{p}^{*} v_{p, p^{\prime}} b_{p^{\prime}}
$$

where $\Lambda$ is the finite volume extent of the system, $\omega_{p}$ is the energy of a free electron of momentum $\mathrm{p}, a_{s, p}^{*}, a_{s, p}$ are the creation and annihilation operators for a spin $s$ electron of momentum $p, b_{p}^{*}=a_{\uparrow, p}^{*} a_{\downarrow,-p}^{*}$ is the creation operator for the Cooper pair; $b_{p}^{*} v_{p, p^{\prime}} b_{p^{\prime}}$ gives the interaction energy between two Cooper pairs. The terms in the Hamiltonian are representative of a fruitful general strategy in condensed matter physics: one treats the interactions between the many elementary constituents of a system as stable particles, and then posits that the interactions between these stable quasiparticles are of a simple nature. ${ }^{9}$

This formulation of the interaction hides the temperature-dependence of the interaction strength. ${ }^{10}$ A more revealing, although approximate, form of (5) is given through a

\footnotetext{
${ }^{9}$ For more insight, see Kadanoff (2013) or Anderson (1997).

${ }^{10}$ The presence and number of Cooper pairs implicitly depends on the temperature. At low temperatures, electrons will have fewer accessible momentum states, and it is thus more likely that anticorrelated pairs will be found.
} 
Bogoliubov-Valantin transformation of operators

$$
\begin{aligned}
& \gamma_{\uparrow, p}=\left(\Delta_{p}^{*} a_{\uparrow, p}+\left[E_{p}-\omega_{p}\right] a_{\downarrow,-p}^{*}\right) / D_{p} \\
& \gamma_{\downarrow, p}=\left(\left[\omega_{p}-E_{p}\right] a_{\uparrow,-p}^{*}+\Delta_{p}^{*} a_{\downarrow, p}\right) / D_{p},
\end{aligned}
$$

where $\Delta_{p}$ is to become the order parameter for the theory, and satisfies the self-consistency equation

$$
\Delta_{p}=-\sum_{p^{\prime}} v_{p, p^{\prime}} \frac{\Delta_{p^{\prime}}}{2 E_{p^{\prime}}} \tanh \left(\frac{1}{2} \beta E_{p^{\prime}}\right),
$$

and

$$
\begin{aligned}
& E_{p}=\left(\omega_{p}^{2}+\Delta_{p} \Delta_{p}^{*}\right)^{\frac{1}{2}} \\
& D_{p}=\left(\left[E_{p}-\omega_{p}\right]^{2}+\Delta_{p} \Delta_{p}^{*}\right)^{\frac{1}{2}} .
\end{aligned}
$$

The new, approximate Hamiltonian then takes the form

$$
\tilde{H}_{\Lambda}=\sum_{p, s} E_{p} \gamma_{s, p}^{*} \gamma_{s, p}
$$

and includes an explicit temperature dependence via $\beta=(k T)^{-1}$ in the order parameter $\Delta_{p}$. The original authors claimed that the approximate and original Hamiltonians coincide in the limit of infinite volume $\Lambda \rightarrow \infty$, so that (8) is an exact expression at the thermodynamic limit (p. 1180). ${ }^{11}$

For most temperatures, $\Delta=0$ is the energetically favourable solution, and there the energy spectra of $H$ and $\tilde{H}$ coincide. However, there is a critical temperature $T_{c}=1 / k \beta_{c}$ below which a solution $\Delta \neq 0$ becomes more energetically stable, and it is this phase that corresponds to the superconducting phase. In this case, there is a degenerate set of lowest energy states, each of which has a fixed phase, breaking the global $U(1)$ symmetry. (8) provides a more explicit description of the broken symmetry, which is marked by the energy gap $\Delta \neq 0$ between the superconducting ground states and the excited 'normal' phase. In this regime electrons couple to form Cooper pair bosons. Cooper pairs are permitted to collectively condense into the energetically favoured superconducting ground state in virtue of being bosons: fermions are prohibited from occupying identical states by the Pauli exclusion principle. ${ }^{12}$ The finite energy gap $\Delta$ inhibits excitations out of the superconducting ground state. This amounts to a non-negligible fraction of the superconductor's electrons occupying a single state, resulting in a macroscopic quantum system.

\footnotetext{
${ }^{11}$ At the thermodynamic limit, one may worry that we are somehow getting different physics by reshuffling the degrees of freedom from the form of (5) to (8), while the two are supposed to describe the same physical situation. Similar worries are shared by philosophers examining the Higgs mechanism. In the case of the BCS model, these issues were resolved by Haag (1962) (according to Emch (2007, p. 1123)). The fact that the approximation here becomes exact in the thermodynamic limit is a fact unique to BCS theory, finding (as far as we are aware) no analogue in QFT.

${ }^{12}$ As Kadanoff (2013) emphasizes, BCS disavow the connection with Bose-Einstein condensates. See his Sec. 3.2
} 
In 1959 Gor'kov derived the GL equations from the BCS equations for systems near the critical temperature. He related the two sets of equations by setting the collective wave function and order parameter $\psi$ in the GL model to be proportional to the order parameter $\Delta$ in the BCS model (Gor'kov, 1959, p. 1366). The derivation entails that $m^{*}$ in $D_{i}$ (eq. $(2))$ is the mass of the Cooper pair $2 m$ and that the $e^{*}$ is the charge of the Cooper pair 2e. For our purposes of analyzing the analogies to EW SSB, the main focus will be the GL and BCS equations. We will thus treat the two models separately; the fact that (given suitable background assumptions) the BCS equations entail the GL equations is incidental to our analysis. However, for the purpose of explaining the phenomena of superconductivity, Kadanoff emphasizes that both the micro-physical account of the origins of superconductivity supplied by the BCS model and a macroscopic understanding of the quantum wave function $\psi$ as representing the entire condensate of superconducting electrons - and not a single particle - are necessary. With this additional understanding we see that, below $T_{c}$, the Cooper pairs are of sufficiently low energy to collectively condense into the ground state, forming a Bose-Einstein condensate of lowest energy Cooper pairs.

In this ground state, however, the global $U(1)$ gauge symmetry characteristic of electromagnetic interactions is broken, since the Cooper pairs condense to form a phase-rigid system. It was later shown that, for the condensate wavefunction, the phase angle is related to the conjugate momentum in such a way as to keep the wavefunction's velocity gauge invariant (see Kadanoff (2013, p. 818)). This entails a rigidity preventing the spatial variation of the phase in the superconducting state. When an external magnetic field is applied to the superconductor, the rigid phase prohibits coupling within the interior of the superconductor, "pushing" the magnetic field out of the material. This is the explanation for the Meissner effect: external magnetic fields vanish within the superconductor. Beyond a small penetration depth, neither electric nor magnetic fields will be present within the superconductor. The short range of the electromagnetic interaction is often regarded as an indication of the effective mass of a photon upon entering the phase-locked superconductor.

The BCS model is characterized as dynamical by contrast with the GL model. The following features - which are opposed to the features identified in Sec. 3.1 as being characteristic of the phenomenological GL model - mark the BCS model as a dynamical model:

1. causal explanations: ${ }^{13}$ The BCS model provides an explanation in terms of underlying interactions and energy levels that accounts for the phase transition (and accompanying SSB) from a normal state to a superconducting state. This is a causal explanation of superconductivity. The cause of the phase transition is that more energetically favourable states become available for the conducting electrons; when these lower energy ground states become possible, pairs of electrons form Cooper pairs and then condense into the lower energy ground states.

\footnotetext{
${ }^{13}$ Note again Kadanoff's point that explanations of the phenomenology of superconductivity require an understanding of the macroscopic significance of the wavefunction supplied by the GL model as well as the microscopic underpinnings supplied by the BCS model. The point here is just that the BCS model introduced ingredients essential for having an explanation (as opposed to merely a description) of the phase transition.
} 
2. micro-constituents: The model employs an appeal to the configuration of micro-constituents of the superconductor (i.e., photons, phonons, electrons, etc.). In particular, the linchpins of the model are the Cooper pairs, which are composites of two electrons.

3. parameters theoretical inputs: The majority of the explanatory parameters in the theory arise from theoretical, not empirical, considerations. (e.g., masses, energies of the particles)

\subsection{The Abelian $U(1)$ Higgs model}

We will provide a brief overview of the Higgs mechanism in the simpler Abelian $U(1)$ case. $^{14}$ This is a standard approach in both the philosophical literature and physics textbooks because the relevant content remains the same, but we avoid getting bogged down in the lengthy expressions used in the breaking of electroweak $S U(2) \times U(1)$ symmetry. Furthermore, we follow the standard contemporary physics textbook presentation of the Abelian Higgs mechanism. To foreshadow: We include this standard presentation of the Higgs mechanism to make contact with the contemporary philosophy and physics literature, but our historically-informed analysis is in fact based on earlier, gauge-invariant presentations of the Higgs mechanism.

The starting point for the Abelian Higgs mechanism is the Lagrangian

$$
\mathcal{L}=\left(\mathrm{D}_{\mu} \phi\right)^{\dagger}\left(\mathrm{D}_{\mu} \phi\right)-V_{H}(\phi)-\frac{1}{4} F_{\mu \nu} F^{\mu \nu}
$$

where $\phi(x)$ is a complex classical field over spacetime, $F_{\mu \nu}=\partial_{\mu} A_{\nu}-\partial_{\nu} A_{\mu}$ is the electromagnetic tensor, $\mathrm{D}_{\mu}=\partial_{\mu}-i g A_{\mu}$ is the gauge covariant derivative, and $V_{H}(\phi)=\mu^{2} \phi^{\dagger} \phi+\lambda\left(\phi^{\dagger} \phi\right)^{2}$ is the famous Higgs Mexican hat potential. $\mathcal{L}$ is invariant under the gauge transformation $\phi \rightarrow \exp (i \alpha(x)) \phi, A_{\mu} \rightarrow A_{\mu}-(1 / g) \partial_{\mu} \alpha(x)$. For $\mu^{2}>0$, the vacuum (minimum energy, Poincaré invariant solution) is the usual one at $\phi=0$, for which all vacuum expectation values $(\mathrm{VEVs})$ are 0 . However, when $\mu^{2}<0$, the vacuum is characterized by:

$$
\langle 0|\phi| 0\rangle=\frac{v}{\sqrt{2}} ; \quad v=\sqrt{\frac{-\mu^{2}}{2 \lambda}} .
$$

Thus the minimum energy solution is not at $\phi=0$; in fact, there exist a continuous number of possible solutions, corresponding to $\phi^{*} \phi=v / \sqrt{2}$, the circle of minimum energy in the potential $V_{H}(\phi)$.

What interests physicists are excitations to the field near the vacuum energy $v$, since we suppose that these are the only energies (currently) accessible by experiment. So we rewrite $\phi$ in terms of an excitation field $\rho$, such that

$$
\phi(x)=\frac{1}{\sqrt{2}}(v+\rho(x)) e^{i G(x) / v} .
$$

\footnotetext{
${ }^{14}$ This presentation follows Marshak (1993, section 4.4a) in the style of notation used. For different presentations of the same content, see Earman (2003), Martin (2003), or Wuthrich (2012), among numerous others. In particular, we use a polar decomposition for the complex scalar field $\phi$.
} 
This can be thought of as fixing the gauge $\alpha(x)=G(x) / v$; in order to fully fix the gauge, we must also define

$$
A_{\mu} \rightarrow B_{\mu}=A_{\mu}-\frac{1}{g v} \partial_{\mu} G(x) .
$$

Fixing the gauge amounts to selecting one out of the infinite number of possible vacuum states, thereby breaking the gauge symmetry. Plugging in these values, we can rewrite the Lagrangian (neglecting terms cubic or higher)

$$
\mathcal{L}^{\prime}=-\frac{1}{4} B_{\mu \nu} B^{\mu \nu}+\frac{1}{2}(g v)^{2} B_{\mu} B^{\mu}+\frac{1}{2}\left(\partial_{\mu} \rho(x)\right)^{2}-\mu^{2} \rho(x)^{2},
$$

where $B_{\mu \nu}$ is the counterpart of $F_{\mu \nu}$ defined in terms of $B_{\mu} . \mathcal{L}^{\prime}$ is interpreted as containing a massive vector field $B_{\mu}$ with mass $g v$, and an additional scalar field $\rho$, with mass $\sqrt{2}|\mu| . \mathcal{L}^{\prime}$ does not contain $G(x)$, which is interpreted as the massless field associated with the Goldstone bosons. The Goldstone bosons are eliminated through the correct choice of gauge. Thus, a renormalizable theory that started with massless mediating bosons has been converted to a theory involving massive mediating bosons, without spoiling the renormalizability of the theory.

Earman (2002) critiques the role that the choice of the unitary gauge plays in this standard presentation of the Higgs mechanism. The problem is that a gauge symmetry (according to philosophical accounts) is supposed to represent "descriptive fluff," a mere redundancy in the mathematical formalism of QFT. All physically relevant parameters are thus required to be gauge invariant. In particular, whether the dynamical equations describe a massive Higgs boson or a massless Goldstone boson should not depend on the choice of gauge. Earman thus rejects the "just-so stories" that particles gain mass "by eating the Higgs field" (2002, p. 1239). In order to determine the correct physical interpretation of the Higgs mechanism, he therefore demands a gauge invariant presentation. Morrison (2003), Smeenk (2006), Struyve (2011), and Lyre (2008) (among others) offer various approaches to this problem.

The issues taken up in this paper are for the most part orthogonal to these issues pertaining to the choice of gauge. One reason for this is historical circumstances. Struyve (2011) points out that the early treatments of the Abelian Higgs model in Higgs (1966) and of the non-Abelian Higgs model in Kibble (1967) were in fact gauge invariant. Struvye reviews Higgs' 1966 gauge invariant presentation of the Abelian Higgs model. According to Struyve, the presentation of the Abelian Higgs model rehearsed above with the appeal to the unitary gauge was first employed by Weinberg as a simpler way of obtaining Kibble's results, and was then picked up in the textbook literature (p. 226). The historical period in which analogies to the BCS model influenced the formulation of the EW component of the Standard Model was c.1960-6, when the gauge invariant presentations of the Higgs mechanism were current. Moreover, most of the analogies to superconductivity are based on the initial Lagrangian (9) and other expressions involving $\phi(x)$ rather than the Lagrangian $(13)$ and $\rho(x) .{ }^{15}$ For the

\footnotetext{
${ }^{15}$ An exception is the discussion of the physical interpretation of the Higgs field in Sec. 5.6. However, as Struyve stresses, the gauge invariant presentation of the Abelian Higgs model results in the same effective Lagrangian, including fields with the same masses (p. 230).
} 
purposes of analyzing the historically important analogies between the BCS and Higgs models, details related to how SSB is formally implemented are not relevant. We will return to the question of how analogies to superconductivity inform the interpretation of the popular 'eating' metaphor for mass acquisition in Sec. 5.5.

\section{Formal analogies between the Higgs mechanism and superconductivity}

The types of analogies (and disanalogies) that obtain between the Higgs model and the two models of superconductivity will be evaluated in detail below. As an aid to keeping track of the horizontal relations, we provide three tables highlighting the relevant similarities. Formal analogies will be examined in this section and potential material and physical analogies will be scrutinized in the next section.

Table 1: Analogies between superconductor models and the Higgs model

\begin{tabular}{|l|l|}
\hline Superconductor models & Higgs model \\
\hline$U(1)$ broken (global) gauge symmetry group & $\begin{array}{l}S U(2) \times U(1) \text { broken (local) gauge symme- } \\
\text { try group }\end{array}$ \\
\hline $\begin{array}{l}\text { (limited-range) photon with effective mass } \\
\text { (two transverse components) }\end{array}$ & massive $W, Z$ bosons \\
\hline $\begin{array}{l}\text { plasmon with massive longitudinal compo- } \\
\text { nent }\end{array}$ & massive Higgs boson \\
\hline no analogue & massless photon \\
\hline
\end{tabular}

Table 2: Analogies between the GL model and the Higgs model

\begin{tabular}{|l|l|}
\hline GL model & Higgs model \\
\hline $\begin{array}{l}\text { free energy density of superconducting state } \\
\mathcal{F}_{s}\end{array}$ & Lagrangian $\mathcal{L}$ \\
\hline$a|\psi(\mathbf{x})|^{2}+\frac{b}{2}|\psi(\mathbf{x})|^{4}$ & $V_{H}=\mu^{2}|\phi(x)|^{2}+\lambda|\phi(x)|^{4}$ \\
\hline $\begin{array}{l}\text { collective wave function for superconducting } \\
\text { electrons } \psi(\mathbf{x}) \text { as the order parameter }\end{array}$ & $\begin{array}{l}\text { scalar particle field } \phi(x) \text { as the order param- } \\
\text { eter }\end{array}$ \\
\hline$T$ & no analogue \\
\hline
\end{tabular}

Table 3: Analogies between the BCS model and the Higgs model

\begin{tabular}{|l|l|}
\hline BCS model & Higgs model \\
\hline energy gap $\Delta$ as the order parameter & $\begin{array}{l}\text { vacuum energy }\langle 0|\phi| 0\rangle \text { as the order param- } \\
\text { eter }\end{array}$ \\
\hline composite Cooper pairs & no analogue \\
\hline$T$ & no analogue \\
\hline Anderson-Bogoliubov collective mode & Higgs boson \\
\hline no analogue & Higgs field \\
\hline
\end{tabular}

Without even entering into the details, it is apparent that the Higgs model is more closely analogous to the GL model than the BCS model: the composite Cooper pairs play a pivotal 
role in the dynamical account of SSB offered by the BCS model and there is no analogue of the formation of Cooper pairs in the Higgs model; in contrast, there are analogues for all of the load-bearing elements of the GL model in the Higgs model (including, most importantly, the collective wave function). As we work through the analogies, it will also be helpful to bear in mind that some of the vertical relations in the dynamical BCS model are causal relations, while the vertical relations in the phenomenological GL model do not include causal relations. As a result, there is the potential for material analogies between the BCS and Higgs models, but not between the GL and Higgs models. These two circumstances supply motivation for alternatives to the Higgs model known as dynamical symmetry breaking models. The analogy between the Anderson-Bogoliubov collective mode and the Higgs boson came to light only recently, and will be discussed in Sec. 5.6.

\subsection{Basic formal analogies to the Higgs model common to the GL and BCS models (Table 1)}

As already mentioned, the possibility of an analogous mass gain for gauge bosons in particle physics motivated a close examination of the BCS model. This analogy predates the construction of a model of SSB including the Higgs boson and the Higgs field. It is clearly spelled out in Anderson (1963), which predates Higgs' 1964 paper and does not contain any details of the Higgs mechanism or Higgs potential (and no mention of the Higgs boson).

Both the Higgs mechanism and SSB in models of superconductivity involve the breaking of a gauge symmetry. Electromagnetism and Yang-Mills theories are the paradigms for theories with gauge symmetries. A gauge symmetry is an internal symmetry of the equations of motion. This means that the dependent variables in the Lagrangian or Hamiltonian are invariant under a symmetry group. Note that this definition includes both global and local gauge symmetries. A local symmetry group is parametrized by real functions of space(time) variables and a global symmetry group is not dependent on the space(time) variables. According to the standard accounts presented above, the superconductivity models represent the breaking of the global $U(1)$ gauge symmetry while the Higgs model represents the breaking of a local gauge symmetry. ${ }^{16}$ While this is a formal disanalogy between the two cases

\footnotetext{
${ }^{16}$ Morrison (2012) draws on a presentation of SSB in superconductors in Weinberg (1986) that identifies local $U(1)$ as the broken symmetry. Interestingly, Weinberg's approach to superconductivity is the converse of ours: he aims to "help those who like myself are more at home at high energy than at low temperature, to appreciate the lessons of superconductivity" by reading the particle physics treatment of SSB back into superconductivity, while we aim to trace the flow of SSB ideas in the other direction, from superconductivity into particle physics (p. 43). More specifically, Weinberg derives a Lagrangian by introducing a NambuGoldstone field and running an analogue of the gauge argument surveyed in Sec. 3.3. The derived Lagrangian differs from both the GL and BCS Lagrangians. (However, as Weinberg notes, many of his argumentsincluding the ones cited by Morrison - are adapted from standard presentations of the GL model (p. 46). Morrison's conclusion that phenomenological properties of superconductors are emergent in virtue of following from SSB independently of the details of the microphysics (i.e., Cooper pairing in the BCS model) can thus be supported by appealing to the GL model.) It is interesting to note that Weinberg himself seems to recognize that he is relying on a formal analogy between particle physics and superconductivity that is accompanied by salient physical disanalogies (see footnote ${ }^{*}$ ) on p.45 and the discussion on pp.52-53).
} 
that is important for understanding some aspects of the models, it is not important for tracing the historical analogies that we want to highlight in this paper. Note also that one can start with an arbitrary symmetry group and break all or part of it. The number of massless bosons that remain is equal to the number of generators of the final symmetry group. For the physically relevant electroweak symmetry breaking, local $S U(2) \times U(1)$ is broken, leaving a residual $U(1)$ symmetry group.

In Hesse's classification, the broken gauge symmetry is a formal analogy because the concept of local gauge symmetries is highly dependent on the shared theoretical framework - in which the vertical relations are mathematical relations - employed by the models of superconductivity and the Higgs model. The shared mathematical structure is the infinite-dimensional symmetry groups representing local symmetries, and the physical interpretations of this formalism may differ in the two domains.

There is a formal disanalogy accompanying this formal analogy: different symmetry groups are broken. However, this is an irrelevant dissimilarity because the Abelian Higgs model in which the $U(1)$ symmetry is broken has the same essential features.

\subsection{Formal analogies between the GL model and the Higgs model (Table 2)}

Many physicists and historians have noted the explicit formal similarity between the Higgs potential and the GL equation. We identify three main formal analogies.

(a) In both instances the desired result is a symmetric Taylor expansion about some nonzero value of the order parameter, and this is why the free energy expression (2) has the same form as the Higgs potential (Table 2, row 3)

$$
V_{H}(\phi)=\mu^{2}|\phi|^{2}+\lambda|\phi|^{4}
$$

Coefficients multiplying odd powers of the order parameter are set to zero to ensure symmetry, and expansion to the fourth order is the lowest order expansion that leads to SSB. In the case of the Higgs mechanism, this is also the largest renormalizable interaction term. Many of the formal similarities between the GL model and the Higgs mechanism arise due to this formal similarity. Though this "Mexican hat" form is shared by the two theories, the terms arise in the context of parameters with different physical interpretations: in the GL model these terms factor into the free energy density of the superconducting state, $\mathcal{F}_{s}$ defined over space, while in the Higgs mechanism they arise in the Lagrangian $\mathcal{L}$ as the form of an external potential over spacetime (Table 2, row 2). Since the mathematical form of the free energy density (GL) and the external potential (Higgs) is the same, both only result in a non-zero order parameter when the coefficient in multiplying the $|\phi|^{2}$ or $|\psi|^{2}$ term is negative. The fact that the similarity of the equations does not stem from some physical or material analogy is evidenced by the origin of the "Mexican hat" terms. In both models there is no attempt to explain the causal-dynamical origin of the terms; instead, the expression is added by hand in order to force SSB. The "Mexican hat" terms are the simplest terms that give rise to the desired result - critical point phase symmetry breakdown in the GL model and avoidance of Goldstone's theorem in the Higgs mechanism. 
(b) In both models, the order parameter is represented by a field parameter $\phi(x)$ or $\psi(\mathbf{x})$. (There is again a difference in physical interpretation: in the GL model, $\psi(\mathbf{x})$ represents a collective wavefunction for the superconducting electrons in the metal, while in the Higgs mechanism, $\phi(x)$ represents some elementary scalar field that interacts with the gauge field.) A formal difference is that $\psi(\mathbf{x})$ is an ordinary function in non-relativistic quantum mechanics and $\phi(x)$ is an operator-valued field (or, more rigorously, an operator-valued distribution). Thus, the maps $\phi$ and $\psi$ have different domains, images, and symmetry groups. In both cases, the symmetry breaking occurs when the energy of the system is locally minimized at $|\phi|=$ constant, $d \phi=0$. This is a consequence of the formal identity of the potential (Higgs) and free energy density (GL), but bears being made explicit.

(c) Most importantly for dynamical accounts of the Higgs mechanism, both models provide a surface account of the processes they describe: the GL model is explicitly phenomenological, and there exists a "micro-theory" - the BCS theory - to explain the phenomena modelled by Ginzburg and Landau, while the Higgs mechanism does not explain the form of the Higgs potential, nor does it provide a causal "micro-theory" akin to the BCS model. This has led physicists to pursue a dynamical model of SSB in the EW theory. We believe this motivation is ill-founded, and discuss this issue in Sections 5.4 and 6 .

Though the formal similarities are plentiful, there are some relevant disanalogies. For example, the GL model contains a temperature-dependent expression for the order parameter, which is found to vanish above the critical temperature. No temperature-dependence is given in the Higgs mechanism. This lack of external control of SSB is noted in Table 2 and discussed below. In (a), (b), and (c) above, the similarities are strictly formal: the same mathematical expression is used in the Lagrangian of the Higgs mechanism as the free energy density of the GL model, in both cases a gauge symmetry is broken, and in both cases the order parameter is given by a field variable. There are fundamental differences in physical interpretation accompanying the formal analogies. Without further analysis, then, it is safe to say that the analogy between the GL model and the Higgs mechanism is obviously a formal one. The vertical relations are similar simply because both models rely on the same mathematical structure. After all, what else could it be but a formal analogy? There is very little in the way of physical or causal content to the GL model that could supply a basis for physical or material analogies to the Higgs mechanism.

\subsection{Formal analogies between the BCS model and the Higgs model (Table 3)}

The formal analogies between the Higgs model and the BCS model are more superficial than those between the Higgs model and the GL model, but formal analogies nevertheless exist. For example, both models are obtained by rewriting their fundamental dynamical equation (Lagrangian in the case of the Higgs mechanism, Hamiltonian in the case of BCS theory) in terms of some sort of approximation, which amounts to introducing different fields from those we started with. The Higgs mechanism deals with small excitation fields around the minimum potential value, while BCS theory deals with the Bogoliubov-Valantin transformed fields. 
Though this similarity is worth noting, there is more to be said. The approximation used in BCS theory resulting in a broken gauge symmetry is only approximate short of the thermodynamic limit. This is an important point to stress, since equations (5) and (8) are physically and mathematically equivalent (the latter in a relevant sense, see Emch (2007, section 5.1)) in the limit of an infinite superconductor. This is not so for the Higgs mechanism: the approximation of small excitations near the vacuum state already arises in the context of infinite degrees of freedom, and never becomes exact. It is instead justified on the grounds that accessible experimental energies are very small, and so higher order fluctuations are negligible.

Both models also contain order parameters to mark SSB. In the BCS model this is the energy gap $\Delta$, which is comparable to the vacuum expectation value of the initial scalar field $\langle 0|\phi| 0\rangle$ in the Higgs mechanism. These physically different terms play an analogous role in the formalism of SSB: both serve as a litmus test for broken symmetry. In Hesse's classification, the similarities between BCS SSB and the Higgs mechanism are primarily similarities of vertical relations: the fact that both mechanisms involve approximations is hardly more than a methodological similarity, since most models employ some sort of mathematical approximation technique.

\section{$5 \quad$ Prospects for material or physical analogies}

Given the fact that there exist formal analogies between the Higgs and both the BCS and GL models of superconductivity, we turn to the prospects for deeper material and physical analogies. Recall that material analogies map vertical relations in the source and target domains that are causal relations of the same type; physical analogies have horizontal relations that rely on physical similarities between the domains. Material or physical analogies would support the transfer of aspects of the physical interpretation of SSB in superconductivity models to SSB in the Higgs model. Material analogies are only possible between the BCS and Higgs models because the GL model is phenomenological. There is the potential for physical analogies with either the BCS or GL models. We argue that the analogies between the BCS and GL models and the Higgs model are neither material nor physical because they are accompanied by fundamental physical dissimilarities.

\subsection{Physical disanalogy: SSB in the Higgs model is not a temporal process}

A crucial physical disanalogy between the Higgs mechanism and both the GL and BCS models reveals itself in the physical interpretations of $x$ and the horizontal analogue $\mathbf{x}$ (see Tables 2 and 3): $x$ in the Higgs model represents spacetime, whereas $\mathbf{x}$ in the models of superconductivity represents space. The collective wave function and states in the BCS and GL models are defined on space; the fields and states in the Higgs model are defined on spacetime. Relatedly, the analogue of the free energy (GL) is the Lagrangian (Higgs). That there is an analogical mapping from space in the superconductivity models to spacetime in 
the Higgs model would make it difficult to introduce a compatible analogical mapping from time in superconductivity to time in the Higgs model.

In fact, under the analogical mapping set out in Tables 1-3, temporal processes in the superconductivity models do not correspond to temporal processes in the Higgs model. A temporal process is a process in which a system undergoes a transition from state ${ }_{1}$ at $t_{1}$ to state $_{2}$ at $t_{2}$. In the models of superconductivity, the temporal process of special interest is that in which a superconductor undergoes a transition from a symmetric ground state at $t_{1}$ to a ground state in which symmetry is broken at $t_{2}$. The ground states are the states with lowest energy, so are picked out by the Hamiltonian (or Lagrangian). The free energy in the GL model (Eq. (2)) and the Hamiltonians in the BCS model (Eq. (5) or (8)) are both temperature-dependent and thus are time-dependent. In contrast, the corresponding 'transition' in the Higgs model is not a temporal process. For example, in the Abelian Higgs model there is no corresponding temporal process in which the system undergoes a transition from a symmetric vacuum state $|0\rangle$ at $t_{1}$ to a vacuum state in which symmetry is broken $\left|0^{\prime}\right\rangle$ at $t_{2}$. A vacuum state is also the lowest energy state, so is also picked out by the Lagrangian (or Hamiltonian). The Lagrangian for the Abelian Higgs model (Eq. (9)) is time-independent. The symmetric vacuum state $|0\rangle$ for the Lagrangian (9) with some fixed mass $\mu\left(\mu^{2}>0\right)$ is a global state (i.e., defined over all of spacetime, not at a time). The vacuum state with broken symmetry $\left|0^{\prime}\right\rangle$ for the Lagrangian (9) with some other fixed value $\mu^{\prime}\left(\left(\mu^{\prime}\right)^{2}<0\right)$ is also a global state. Since $|0\rangle$ and $\left|0^{\prime}\right\rangle$ are both global states and describe different systems with different masses $\mu$ and $\mu^{\prime}$, there is no temporal process between $|0\rangle$ and $\left|0^{\prime}\right\rangle$.

As Earman points out, considerations about whether SSB is a temporal process in the Higgs model are closely tied to modality. He argues that "as long as the system of interest is closed, there is no temporal evolution involved in spontaneous symmetry breaking in QFT since every physically relevant state is asymmetric with respect to the symmetry of the Lagrangian" (Earman (2003, p.337), Earman (2004, p.187)). For example, for the Abelian Higgs Lagrangian with fixed $\mu^{\prime}\left(\left(\mu^{\prime}\right)^{2}<0\right)$ all of the physically possible states-including the vacuum states lying on the rim of the Mexican hat-are asymmetric. The symmetric vacuum state is only physically possible in a different possible world with dynamics described by a different Lagrangian with fixed $\mu\left(\mu^{2}>0\right)$. Earman also contrasts this feature of SSB in QFT with SSB in classical mechanics and non-relativistic quantum mechanics. The underlying reason for the difference between QFT and models of superconductivity constructed using non-relativistic quantum mechanics is not the asymmetric Mexican hat structure of the states - which the Higgs model shares with the GL model-but that the Mexican hat potential appears in the time-dependent expression for free energy in the GL model and in the time-independent Lagrangian in the Higgs model.

Modal differences between the Higgs and superconducting models are also illustrated by the different statuses of the gauge bosons in the two cases. At the time at which the temperature of the superconductor reaches $T_{c}$ and SSB occurs, photons inside the superconductor undergo a transition from being long-range and massless to effectively short-range 
and effectively massive. ${ }^{17}$ Massless and massive are two possible states of the photon in the sense that it is possible for the system to occupy the states at different times. In the Higgs model, the $W$ and $Z$ bosons are massive at all times and there actually never were (or are or are going to be) any massless $W$ and $Z$ bosons. In the physics literature, this modal distinction is marked by the use of the terms state or mode to describe condensed matter systems only, and the application of the term boson to both condensed matter and particle physics systems. Marshak writes that, for global Abelian and non-Abelian groups, the Goldstone theorem guarantees that SSB "gives rise to massless scalar (N-G" ${ }^{18}$ states in non-relativistic many-particle systems and to N-G bosons in QFT, and both condensed matter and particle physics have been enriched by the recognition of the existence of N-G excitations (whether states or bosons!)" (p. 219). States at a time in condensed matter physics are contrasted with boson fields existing at all times in particle physics. (Whether both are species of excitation in some interesting sense will be taken up below.)

A related feature of SSB in superconductivity is that, at one time, photons have different effective masses inside and outside of a superconductor. In contrast, in particle physics, SSB does not entail that there are sub-systems of the universe in which $W$ and $Z$ bosons are massless and others in which they are massive. Anderson notes that there is a related difference in the experiments that can be done to ascertain properties of superconductors and particle physics systems (1963, pp. 440-441). It is possible for a solid state physicist to get outside of a superconductor and impose an external electromagnetic field. This is an experimental means of determining the response of the current to the electromagnetic field. In contrast, in particle physics it is not possible to get outside of the vacuum state, for instance, to impose an external electromagnetic field; the system takes up the entire universe. The only means of experimentally detecting the response of the current to the gauge field in particle physics is to introduce a charged test particle (which, of course, can also be done in superconductors); however, this method does not allow experimenters to directly determine the response function of the current because the test particle induces its own gauge field. In QFT terms, we never find the "bare" properties of the field in question, only the renormalized properties.

Put another way, time is an external parameter in the superconductivity models, but not the Higgs model. Temperature is also an external parameter. The phase transition from a 'normal' metal to a superconducting metal can be induced by lowering the temperature below $T_{c}$. The lack of an analogue of $T$ in the Higgs model has already been noted. Presuming that the universe is a closed system, there is no external parameter analogous to temperature that induces a symmetry-breaking or -restoring phase transition.

The fact that superconductors are subsystems has further implications for the empirical evidence available to support theories of SSB in superconductivity versus SSB in particle physics. We have good evidence that the underlying symmetry in BCS theory is a physical symmetry: quantum electrodynamics (QED) is thought to govern all electromagnetic phenomena, of which superconductivity is a subset, and QED is manifestly $U(1)$ gauge invariant.

\footnotetext{
${ }^{17}$ This is clearly an idealization. It takes time for the system to reach a new equilbrium state.

${ }^{18} \mathrm{Nambu}-G o l d s t o n e$
} 
As Kosso points out, "[g]iven the antecedent justification for believing in gauge symmetry of the interaction, there is good reason to look for a mechanism that breaks the symmetry in the superconductor" (2000, p. 365). In contrast, in the electroweak theory, all observable phenomena occur with the symmetry already broken. There are no analogous empirical grounds for inferring that there is an underlying symmetry that gets broken in this case.

Castellani (2003, pp. 321-2) urges that two senses of 'symmetry breaking' be kept distinct: (i) "the ['dynamical'] process by means of which the considered symmetry is broken" and (ii) "a broken symmetry situation (or simply the fact that a symmetry is not there)" 19 . In terms of this distinction, our conclusion is that both senses of symmetry breaking are applicable to the models of superconductivity, but that only the latter is applicable to the Higgs model.

Finally, we again stress that the conclusions in this section are restricted to physical analogies between superconductor models and the Higgs model component of the Standard Model. The analogies to SSB in superconductors which inspired the Higgs model do not establish that the corresponding SSB in the Higgs model is a temporal process and do not identify an analogue of temperature in the Higgs model. Naturally, the fact that the analogy with superconductivity which guided the introduction of SSB does not extend to time or temperature does not entail that it is impossible to formulate a particle physics model in which SSB is a temporal process or in which there is a temperature variable. Here are three ways in which such a project could proceed:

1. model an open subsystem using QFT and introduce one or more external variables

2. introduce an explicit time-dependence in the mass parameter $\mu$ in the Lagrangian ${ }^{20}$

3. introduce a temperature variable into QFT (e.g., finite temperature field theory (Le Bellac, 2000))

All of these strategies involve the development of new physics that builds on or replaces the Higgs model. Of course, any model constructed along one of these lines would have to be subject to empirical test to determine whether it is a better candidate for representing the actual world than the Higgs model. For philosophers, the important point is that philosophical analysis of a temporal process of SSB in particle physics would have to attend to one of these alternative models, and not to the Higgs model component of the Standard Model. Moreover, the analogies to superconductivity that inspired the introduction of SSB

\footnotetext{
${ }^{19}$ Castellani adds "the result of a symmetry-breaking process" to her characterization, which is of course not applicable to SSB in the Higgs model, on our understanding.

${ }^{20}$ This is a variant on Earman's example, in analogy with the example in classical mechanics of a bead on a hoop which has a ground state that exhibits spontaneous symmetry breaking when the angular velocity is above a critical value, of "a parameterized family of Lagrangians for a field such that there is a symmetry of the Lagrangian that is unitarily implementable for the quantized field until, but not after, the parameter attains a critical value" $(2004,187)$. (The example is presented in the algebraic framework for QFT- see Sec. 5.2 below.) Earman remarks that "[a] request for a dynamical explanation of the change of value in the parameter is perfectly in order" (ibid). We agree, but point out that (a) this is not an example of "ubiquitous" symmetry breaking in Earman's sense and (b) the Abelian and non-Abelian Higgs models are not models in which the critical parameters in the Lagrangian vary.
} 
into particle physics in the first place do not furnish a basis for the new physics in these models - the inspiration for the new physics must come from elsewhere.

\subsection{Modality and SSB in algebraic QSM and QFT}

The differences in the roles played by time in QSM and QFT are particularly transparent in the algebraic formulations of the theories. For a brief introduction see Earman (2002, 2003) and for a comprehensive account see Ruetsche (2011). The algebraic framework casts $\mathrm{SSB}$ in QSM and in QFT in the same mathematical form: ${ }^{21}$ "a symmetry $\alpha$ is broken in the [algebraic] state $\omega$ on [algebra] $\mathfrak{A}$ just in case $\alpha$ is not unitarily implementable on $\omega$ 's GNS representation" (Ruetsche, 2011, p. 300).

Time already enters into the axioms for QSM and QFT in different ways. The HaagKastler axioms for QFT associate algebras of observables with (open bounded) regions of spacetime (see, e.g., Ruetsche (2011, Sec. 5.2)). Time translations are automorphisms of the algebra. Algebraic axiomatizations of QSM associate algebras of observables with (open bounded) regions of space (Ruetsche, 2011, Sec 5.4). As a consequence, treating time evolution in QSM can be far from trivial, especially in the thermodynamic limit (Ruetsche, 2011, pp. 284-7). The different roles of time flagged in the preceding section are thus not specific to models that exhibit SSB, but entirely generic differences between QFT and QSM.

Algebras of observables are defined over space in QSM and spacetime in QFT because the former is a non-relativistic theory and the latter is a relativistic one. Relativity could also be the underlying reason for the mapping of space in non-relativistic models of superconductivity to spacetime in relativistic Higgs models. The algebraic axiomatization of QFT furnishes a useful framework in which to isolate the consequences of relativity. The ground states in the superconductivity models and the vacuum states in the Higgs models are all lowest energy states. In addition, a vacuum state in a QFT is Poincaré invariant. Ruetsche argues that - in both the abstract algebraic representation and a concrete Hilbert space representation constructed according to the GNS prescription-natural ways of interpreting the operators $A(\mathcal{O})$ as representing operations performed in the spacetime region $\mathcal{O}$ fail (pp. 110-111). The Vacuum and Primitive Causality axioms, which impose requirements of relativistic spacetime, are responsible. In particular, non-relativistic QSM admits a Heisenberg picture-style interpretation in terms of time-independent algebraic states $\omega$ and a time-dependent algebra of operators when there is an an automorphism $\alpha_{t}$ representing time translation: ${ }^{22} \quad \alpha_{t}(A)$ is the time-evolved counterpart of $A$. Ruetsche argues that algebraic QFT does not admit such a Heisenberg picture-style interpretation (p. 115). The problem is that in QFT the analogous automorphism $\alpha_{t}{ }^{23}$ cannot be interpreted as representing time

\footnotetext{
${ }^{21}$ Both QSM and QFT exhibit SSB in what Ruetsche labels the individual sense (defined here). Whether QFT SSB also takes the form of SSB in the decompositional sense, as does QSM, is an open question (Ruetsche 303-304 and Chapter 14).

${ }^{22}$ In some cases, there is no automorphism of the algebra that implements the dynamics (Ruetsche, 2011, p. 284ff.).

${ }^{23}$ Details (for the example of Klein-Gordon theory in Minkowski spacetime and spacelike hypersurfaces $\Sigma_{1}$ and $\Sigma_{2}$ ): "Where $\Sigma_{1}$ and $\Sigma_{2}$ are Cauchy surfaces and $\iota$ an identity map between them, [ $\left.\alpha_{t}\right]$ is the
} 
evolution. Where $\Sigma_{1}$ and $\Sigma_{2}$ are spacelike hypersurfaces (and also Cauchy surfaces), $\alpha_{t}$ does not time evolve an element of algebra $\mathfrak{A}\left(\Sigma_{1}\right)$ to an element of algebra $\mathfrak{A}\left(\Sigma_{2}\right)$. This interpretation is again blocked by the Primitive Causality axiom, which entails that any state $\omega$ on $\mathfrak{A}\left(\Sigma_{1}\right)$ is also defined on $\mathfrak{A}\left(\Sigma_{2}\right)$. If $\alpha_{t}$ were to represent time evolution of operators in the Heisenberg picture-style representation, then it would represent time evolution of states in the counterpart Schrödinger picture-style representation. However, $\alpha_{t}$ cannot represent the time evolution of states because $\omega$ is a global state; if $\omega$ represents the state on $\mathfrak{A}\left(\Sigma_{1}\right)$, then $\omega \circ \alpha_{t}$ does not represent the state on $\mathfrak{A}\left(\Sigma_{2}\right)$ because (by Primitive Causality) the state on $\mathfrak{A}\left(\Sigma_{2}\right)$ is also $\omega$ (p. 115). Of course, this does not entail that there is no time evolution in QFT. The point is that time evolution is represented in QFT by tying algebras of observables to spacetime regions, and not by an automorphism of the algebra; in contrast, non-relativistic QSM (or QM) ties algebras to regions of space, which permits time evolution to be represented by an automorphism of the algebra. Any relativistic quantum theory that seeks to represent SSB as a temporal process in one of the ways sketched at the end of Sec. 5.1 is either going to have to work within these constraints of QFT or else revise the principles underlying QFT.

The analysis of the differences in the roles of time and modality in the preceding section also has implications for Ruetsche's Coalesced Structures Argument. In QSM, the physical states that induce the unitarily inequivalent representations are equilibrium states; in QFT, the physically relevant states are the vacuum states. Ruetsche argues that, in order to provide an adequate explanation of phase transitions, a theoretical framework for QSM must have the capacity to represent multiple equilibrium states in different phases at the same temperature, since it is possible for one system at the critical temperature to be in one of a number of equilibrium states. This is a central plank of her Coalesced Structures Argument. She tentatively suggests that the Coalesced Structures Argument also applies to QFT. Our analysis of SSB in QFT highlights a difference in modal structure that would need to be taken into account by any attempt to extend the Coalesced Structures Argument to QFT. While in QSM the multiple equilibrium states at a critical temperature are all copossible in that they are accessible to a system at a time, this is not so for QFT, in which the global vacuum states related by the broken symmetry do not represent states accessible to a system at a time. One QSM system can occupy different equilibrium states at different times, and may even exist in a superposition of equilibrium states at a critical temperature; one QFT system cannot occupy different global vacuum states at different times and cannot be in a superposition of global vacuum states. Moreover, distinct global vacuum states are not even accessible to one system at different times. In addition, note that this difference in physical interpretation is unrelated to issues about how to interpret a spontaneously broken gauge symmetry (see Ruetsche (2011, pp.333-5)), in keeping with our separation of the two issues.

automorphism of [the Weyl algebra] $\mathfrak{W}$ corresponding to the symmetry of the classical solution space that maps a solution with initial data $\phi$ on $\Sigma_{1}$ to the solution whose initial data on $\Sigma_{2}$ is the first solution's $\Sigma_{1}$ initial data, transferred to $\Sigma_{2}$ by the identity map $\iota "$ (Ruetsche, 2011, p. 115). 


\subsection{Material analogy undermined}

SSB in superconductors is a temporal process. Both the BCS and GL models offer descriptions of temporal processes that include phase transitions during which symmetry is spontaneously broken. The BCS model moreover offers a description of a causal process during which symmetry is spontaneously broken. A causal process is a temporal process satisfying some additional requirements. Clearly, since SSB in the Higgs model is not a temporal process, it is not a causal process either. The argument does not depend on details of accounts of causation (including whether retrocausality is admitted); the only relevant feature of causal processes is that they are also temporal processes.

A consequence of the fact that SSB is not a causal process in the Higgs model is that there cannot be material analogies between the BCS model and the Higgs model. Recall that Hesse's core requirement for material analogies is that the vertical relations in both the source and target domains be causal relations of the same type. The BCS model presents SSB as a causal process and offers an explanatory account of the causal relations underlying SSB. However, the SSB analogue in the Higgs model is not a causal process (and thus there is no account of causal relations in the Higgs model). Therefore, the analogies between the BCS and Higgs models are not material analogies. It is possible that there are other physical analogies between the BCS and Higgs models. This possibility will be investigated in the remainder of this section.

\subsection{The dynamical-phenomenological distinction does not carry over to the electroweak theory}

The Higgs model bears a closer formal analogy to the GL model than to the BCS model. In particular, there is no analogue of composite Cooper pairs in the Higgs model. This has inspired the thought that there may be a model of EW SSB that stands in the same relationship to the Higgs model as the BCS model stands to the GL model (i.e., X : Higgs :: BCS : GL). This line of reasoning is a motivation for the program of developing 'dynamical' models of SSB for the EW interaction, to be discussed in Sec. 6 below. Sticking to the Higgs model for the moment, can the Higgs model be considered phenomenological in the same sense as the GL model?

Recall the criteria that we used to classify the BCS model as a dynamical model and the GL model as phenomenological one:

Table 4: Dynamical vs. Phenomenological Models

\begin{tabular}{|c|l|l|}
\hline & Dynamical BCS model & Phenomenological GL model \\
\hline 1 & causal explanations & descriptions \\
\hline 2 & micro-constituents & collections of constituents \\
\hline 3 & theoretical inputs & empirical inputs \\
\hline
\end{tabular}

As we argued above, SSB in the EW theory is not a causal process. Thus, the first distinction is inapplicable to EW theory. It is not merely the case that the Higgs model 
offers a description without offering a causal explanation of SSB, as does the GL model; rather, there is no causal process in the course of which symmetry is broken, so no causal explanation is possible. There is no missing causal explanation for a 'dynamical' model X to supply.

The Higgs field and associated Higgs boson do not describe a collection of constituents, as the Higgs model is generally understood. Recall that the centerpiece of the GL model is the condensate wave function, which represents the collection of superconducting electronsa collection of elementary particles. In contrast, the Higgs boson is usually regarded as a single elementary particle. This standard physical interpretation will be discussed in more detail below, but the present point will stand: the Higgs model cannot be regarded as phenomenological in the sense of describing collective phenomena rather than elementary micro-constituents.

Regarding the third feature, the GL model counts as phenomenological because the constants in the potential are empirically determined, and not predicted by the model (e.g. the penetration depth $d$, and coherence length $l$ ). These are also experimentally and theoretically meaningful parameters that characterize and distinguish different superconductors. One pragmatic virtue of the GL model is that it allows prediction of some empirical parameters when other known values are introduced. The Higgs potential contains unspecified parameters, but few of these are experimentally determinable. As Marshak (1993) notes, $\lambda$ remains an unknown parameter (p. 223). Particle masses that arise as a consequence of the Higgs mechanism are measurable, including the recent discovery of the Higgs boson itself. However, these experiments were notoriously difficult to carry out and served as empirical tests of the model rather than empirical inputs or a measurement of the predicted value of a parameter. Additionally, the EW theory does predict mass ratios, determined by the weak mixing angle $\theta_{W}$. So various aspects are theoretical, while others are empirical. However, the pragmatic virtue of the Higgs mechanism is that it preserves renormalizability of a theory containing massive gauge bosons, not that it determines the actual mass relationships. Criterion 3 is therefore not straightforwardly applicable to the Higgs mechanism.

The phenomenological-dynamical distinction does not apply to SSB in the EW theory. None of the three features that comprise the phenomenological-dynamical distinction in superconductivity are applicable to the Higgs mechanism. There is therefore no reason to believe that there is a physically dynamical model that stands in the same relation to the Higgs model that the BCS model stands to the GL model. That the Higgs model is 'merely phenomenological' cannot therefore serve as a motivation for seeking out a 'dynamical' model of SSB in analogy to the BCS model. Most significantly, there is no sense in which the Higgs model supplies mere descriptions of SSB that cry out for deeper causal explanations. After the GL model and other phenomenological models had been constructed, solid state physicists were motivated to seek out an underlying dynamical model by the desire for a causal explanation for superconductivity phenomenology. The search for 'dynamical' models of SSB in particle physics cannot have the same motivation.

We do not want to suggest that the program of constructing models for the Higgs phenomena by analogy to the BCS model is illegitimate. In the regime of interest near the critical 
temperature, the BCS model recovers all of the predictions of the GL model. As Gor'kov showed, the GL equations are entailed by the BCS equations (under suitable assumptions). As we shall discuss in the next section, an important feature of so-called dynamical models of SSB in particle physics is that their predictions diverge from those of the Standard Model at high energy scales. This program produces alternative Lagrangians to the Higgs Lagrangian, which form the basis for alternative, empirically inequivalent models to the Higgs models. However, these alternatives should not be regarded as dynamical models underlying or explaining the Higgs model.

\subsection{Remarks on mass acquisition}

An eating metaphor is often used in pedagogical presentations of the Higgs mechanism. For example, in Marshak's text, the standard presentation of the Abelian Higgs model rehearsed in Sec. 3.3 above is followed by the explanation that "the 'would-be' Goldstone boson... is manifestly gone ('eaten up') and has been converted into the longitudinal mode of the vector (gauge) field" (p. 220). That is, the initial Lagrangian (9) contains a massless gauge vector field, a Goldstone field that is presumed to be massless, and the final Lagrangian (13) - rewritten with a different choice of gauge - contains a massive gauge vector field and the massive (neutral) Higgs scalar field. ${ }^{24}$ Earman has memorably criticized the eating metaphor that is often used for mass acquisition by gauge bosons in the context of SSB. The objection is that rewriting the Lagrangian in a different gauge cannot cause the gauge vector bosons or the Higgs boson to acquire mass because "a genuine property like mass cannot be gained by eating descriptive fluff, which is just what gauge is" (2002, p. 1239).

The goal of this section is not to investigate this criticism pertaining to gauge invariance, but to point out a different problem with the eating metaphor which is illuminated by consideration of the analogy to superconductivity. Eating is a causal process: eating food at $t_{1}$ causes mass gain at $t_{2}$. We have already argued that SSB in the EW theory is not a temporal process and a fortiori not a causal process. At the intermediate stage of the calculation outlined in Sec. 3.3, Marshak describes the Goldstone field as "the massless 'would be' Goldstone field" (p. 220, emphasis added). This phrase indicates that the eating metaphor should not be read literally as describing a casual process. Nothing can gain mass by eating counterfactual food. The eating metaphor is misleading about the ontological status of mass acquisition in this respect. In order to understand what the eating metaphor is intended to convey, the comparators need to be more carefully characterized. That is, what is the massless 'pre-eating' scenario that is being compared with the massive 'post-eating' scenario?

The impression given by standard presentations of the Higgs mechanism such as Marshak's in Sec. 3.3 is that the comparison is between the initial Lagrangian (9)-interpreted as representing a massless vector gauge boson $A_{\mu}$ with two degrees of freedom and a complex (charged) scalar boson $\phi$ with two degrees of freedom - and the final Lagrangian (13) interpreted as representing a massive vector gauge boson $B_{\mu}$ with three degrees of freedom

\footnotetext{
${ }^{24}$ In his classic presentation of the Higgs mechanism, Coleman employs the eating metaphor in the same way as Marshak (1985, pp.123-124).
} 
and a massive scalar neutral boson $\rho(x)$ with one degree of freedom. Formally speaking, the gauge boson absorbs one of the degrees of freedom of the complex scalar field. The Goldstone bosonic field only appears at an intermediate stage of the manipulation. However, the role played by the Goldstone boson is obscured by questions surrounding how to interpret the choice of gauge. But this is not the thread we will follow here. Instead, we will consider the significance of mass acquisition in the original papers, including the gauge-invariant treatment of the Abelian Higgs model in Higgs (1966).

Before surveying the options canvassed in the original papers, consider the following interpretation of the eating metaphor, which is suggested by the analogy to superconductivity. In superconductors, SSB is a temporal process. Prior to the phase transition, the photon is massless; following the phase transition, the photon has an effective mass. One could attempt to apply the eating metaphor to describe this causal process as follows: the photon gains effective mass by 'eating' the phonon. However, this is not how the eating metaphor is applied in either superconductivity or the EW theory. The eating metaphor does not describe this causal process. Instead, it is used to compare the Higgs model to a counterfactual scenario.

Table 5: Possible interpretations of the eating metaphor.

\begin{tabular}{|l|l|l|}
\hline & $A$ ('pre-eating') & $B$ ('post-eating') \\
\hline $\begin{array}{l}\text { Epistemic } \\
\text { interpretation }\end{array}$ & $\begin{array}{l}\text { massless gauge boson + massless } \\
\text { Goldstone boson + SSB }\end{array}$ & $\begin{array}{l}\text { massive gauge boson + massive } \\
\text { Higgs boson + SSB }\end{array}$ \\
\hline $\begin{array}{l}\text { Higgs 1964/1966 } \\
\text { interpretation }\end{array}$ & $\begin{array}{l}\text { Goldstone's scalar field the- } \\
\text { ory (with massless Goldstone } \\
\text { bosons) }+ \text { SSB }\end{array}$ & $\begin{array}{l}\text { Goldstone's scalar field coupled } \\
\text { to U(1) vector gauge field (with } \\
\text { massive gauge bosons) + SSB }\end{array}$ \\
\hline
\end{tabular}

Here is a survey of viable options for interpreting the objects of comparison in the eating metaphor:

(1) Epistemic interpretation. Scenario A is the set of misconceptions about gauge theories with spontaneously broken symmetry that prevailed in particle physics circa 1960: that gauge bosons are massless and that SSB is accompanied by massless Goldstone bosons. Scenario B is the corrected understanding of the consequences of spontaneously broken symmetry that was introduced by Anderson, Higgs, and others. From our present perspective, scenario A is not a consistent theory. The 'would be' massless Goldstone bosons are included in the mistaken scenario A. There is no physical relationship between scenarios A and B.

(2) Higgs' 1964 and 1966 interpretation with two Lagrangians (see also Higgs (2014, p. 852)). Scenario A is the scalar field theory that Goldstone used to illustrate Goldstone's theorem. The relationship between scenarios A and B is that the Lagrangian for A is identical to the Lagrangian for $\mathrm{B}$ with the couplings for the terms containing the gauge field set to zero. In scenario B, the 'would be' Goldstone boson is the Goldstone boson that would accompany spontaneous symmetry breaking if the couplings for the terms containing the gauge field were set to zero. As Higgs explains, "the longitudinal polarization of the massive vector excitation replaces the massless scalar excitation which would occur in the absence of coupling to the gauge field" (1966, p. 145).

Both options can appeal to the analogy to superconductivity as evidence that Goldstone's 
theorem breaks down in the analogous situations in particle physics. However, further work would have to be done to identify the assumptions of the theorem that fail. For example, neither option specifies a resolution to Earman's trilemma (2002, p. 1239).

Anderson (1963) does not analyze mass gain by comparing different Lagrangians, as Higgs does in his slightly later papers, but he does consider the effects of turning on the electromagnetic interaction. On this approach, scenarios A and B are related by a thought experiment: if the coupling with the gauge field is initially switched off in scenario A, and subsequently switched on in scenario B, then the Goldstone bosons disappear and the vector gauge bosons become massive. In such a thought experiment, we could think of "eating" occurring when the gauge field is switched on. This is clearly a thought experiment; it is not possible to switch on and off the coupling to the gauge field. Anderson approaches the matter from an experimental viewpoint, asking whether there is any way of experimentally determining the mass of the electromagnetic gauge field prior to turning on the electromagnetic interaction. In particle physics, polarization of the vacuum physically reflects the response of the current to the EM field and is represented mathematically by renormalization; in solid state physics, the ground state of the plasma is also dependent on the response of the current to the EM field. Is there a way to experimentally determine the mass of the gauge field and the response function (especially whether it is finite) prior to turning on the EM interaction? In both particle physics and solid state physics, the response function of the current to the EM field cannot be determined directly by introducing a test particle because the test particle introduces its own EM field. In solid state physics, we can get outside the plasma and impose an external electromagnetic field. This is not possible in particle physics because we cannot similarly get outside the vacuum.

In our view, both the epistemic and Higgs 1964/6 interpretations of the eating metaphor are defensible. The main morals (on either interpretation) are that 'eating' is used to compare counterfactual scenarios, not to describe a physical process, and that there were historical reasons for comparing these particular counterfactual scenarios. Mass is gained relative to a counterfactual scenario in which the 'would be' Goldstone bosons and 'would be' gauge bosons are both massless. In the historical context of the early 1960s, this counterfactual scenario was relevant because - from the epistemic perspective - particle physicists mistakenly believed that spontaneously broken gauge theories described massless gauge bosons and massless Goldstone bosons, and no candidate massless, long-range particles had been observed. From the perspective of a focus on the formalism, the counterfactual scenarios presented by Goldstone's theorem and gauge theories without SSB were relevant because, again, each described massless particles. The important question concerning particle mass was not "How do gauge bosons acquire mass?," but "Is it possible for a gauge theory with spontaneously broken symmetry to describe massive gauge bosons?" The former question asks for a dynamical process in which gauge bosons undergo a transition from being massless to massive; the latter asks about the representative capacity of a particular formalism. The latter question captures the use to which the eating metaphor is put in describing the Higgs model: there is a sense in which Yang-Mills theories without massive gauge bosons were important to the historical development of the theory. 
There is another heuristic difference in the roles of mass acquisition in the development of the theories of superconductivity and EW interactions. Though it is the case that, in both BCS theory and the Higgs mechanism, long range force-carrying bosons are converted to short range bosons, the significance of these conversions marks a relevant disanalogy. Massive gauge bosons were necessary to explain the phenomenology of the weak interaction, and the Higgs mechanism was deployed specifically to account for the masses of the weak gauge bosons. On the other hand, it is only an explanatory artifact of BCS theory that the photon becomes massive within the superconductor. Nothing about the phenomenology of the Meissner effect required that photons become massive within the superconductor, only that the magnetic field must vanish. Since the electric field vanishes in a normal conductor without the photons within gaining an effective mass, a massive gauge boson could not have been considered a necessary theoretical underpinning for the Meissner effect.

\subsection{Anderson: "Maybe the Higgs boson is fictitious!"}

Having identified many substantial physical disanalogies between the Higgs model and the formal analogue models of superconductivity, we are now in a position to return to the main question that motivated this paper: do analogies with superconductivity provide any insight into the physical interpretation of the Higgs boson or the Higgs field? ${ }^{25}$ The central physical disanalogies are the following: the formal analogical mappings do not map temporal processes in superconductivity to temporal processes in particle physics, the causal process of SSB in the BCS model is not mapped to a causal process in the Higgs model, the phenomenological-dynamical distinction does not apply to the Higgs model and some unknown model X, and the 'eating' metaphor is applied to describe a counterfactual theory rather than a dynamical process. We have already noted that 'particles' in superconductor models are defined over space and their properties (e.g., effective mass) may change over time. This ontological status is indicated by use of the terms "state" and "mode" interchangeably with particle terminology (e.g., "boson"). In contrast, the particles in the Higgs model are defined over spacetime. They are never referred to as states or modes, only as particles (e.g., "boson"). In this subsection, we will investigate whether Miller's political analogy based on conductors or the historical analogies to the GL and BCS models supplies a plausible physical interpretation for the Higgs field or the Higgs boson. Our conclusion is that the physical disanalogies undermine attempts to export physical interpretations from condensed matter physics to the Higgs model. We conclude by considering how Anderson's recent speculation that the Higgs boson is "fictitious" relates to our analysis.

To set up his political analogy, Miller (1993) explicitly uses an analogy to a conductor, rather than a superconductor, to explain the physical meaning of the Higgs field and the Higgs boson. Presumably, a conductor is chosen because this model is simpler and thus better suited to his pedagogical purpose. In any case, one of the end points of his reasoning is an explanation of why $W$ and $Z$ bosons have mass and photons do not, which as we have seen was a central motivation for the historical superconductivity analogy. Margaret Thatcher

\footnotetext{
${ }^{25}$ For a discussion of the physical interpretations of the vacuum state in the Higgs model and the ground state in the BCS model, see Morrison (2003).
} 
effectively gains mass when she enters a crowd of (presumably Conservative) political party workers because they cluster around her and make it more difficult to move around the room. The analogy with solid state physics is that an electron moving through a crystal lattice of positively charged ions gains effective mass because it attracts the lattice ions. The Higgs field is the analogue of the crowd and the ionic lattice: "[t]he postulated Higgs field in the vacuum is a sort of hypothetical lattice which fills our Universe" (p. 27). Ironically, in Miller's analogy the Higgs boson is analogous to a rumour spreading amongst the political crowd. He explains that "[s]ince the information is carried by clusters of people, and since it was clustering which gave extra mass to the ex-Prime Minister, then the rumour-carrying clusters also have mass." In terms of solid state physics, the analogue is phonons, which are "waves of clustering" that propagate in a crystal lattice and are not due to the presence of negatively charged particles. By analogy, the Higgs boson is "a clustering in the Higgs field" (ibid). There is another irony here: the phonon is not a real particle, but a "quasiparticle'.

Table 6: Miller's political analogy

\begin{tabular}{|l|l|l|}
\hline Politics & Model of conductivity & Higgs model \\
\hline Crowd & Lattice of ions & Higgs field \\
\hline Margaret Thatcher & Electron & W, Z bosons \\
\hline Knot of people surrounding Thatcher & Effective mass of electron & Mass of W, Z bosons \\
\hline Rumor-carrying cluster & Phonon & Higgs boson \\
\hline
\end{tabular}

Contrast the horizontal relations laid out in this table to those in Table 2 (analogies between the GL and Higgs models) and Table 3 (analogies between the BCS and Higgs models). The horizontal relations identified by Miller are different from those drawn historically to either the GL or BCS models. The underlying reason for the discrepancy is that while all three sets of analogies share the same basic initial physical motivation, Miller's analogy is based entirely on this starting point and the historical analogies to the GL and BCS models use the initial physical inspiration as a jumping-off point to develop a formal analogy, as we have seen. The shared inspiration is that the models of conductors and superconductors include effectively massive particles. Miller puts forward the mechanism for effective mass gain in the electrons in the conductor as an explanation for the massiveness of the $\mathrm{W}$ and $\mathrm{Z}$ bosons and the Higgs boson. In contrast, the effectively massive particles in the superconductivity models that motivated the analogies were the gauge bosons and the would-be Goldstone bosons. The formal analogies to the GL and BCS models are centered around the formal role of the order parameter in characterizing SSB. Since Miller's analogy and the historical analogies each identify different sets of horizontal relations, each will have to be evaluated separately on whether it permits the transfer of the physical interpretation from the model of superconductivity (or conductivity) to the Higgs model.

In Miller's analogy, the Higgs field is analogous to the lattice of ions. This literal interpretation of the Higgs field as being a sort of medium is reminiscent of the analogies to fluids that guided the development of electromagnetism in the nineteenth century and the positing of ether as a medium to support the propagation of electromagnetic waves. The analogy to a lattice of ions clearly cannot supply a physical interpretation for the Higgs field - the Higgs 
field is not literally a lattice of charged particles. This interpretation is at odds with the role that the Higgs field is standardly understood to play in the Higgs model. If the Higgs field were itself a collection of particles - like the ether in models of electromagnetism in the nineteenth century - then there would be a further project of classifying these constituent particles in the Standard Model and detecting them. But there is no such project. Within the Standard Model, the Higgs field is interpreted as being associated with the Higgs boson, an elementary particle. The physical interpretation of the Higgs field cannot be based on the fact that there is a charge distribution over the lattice points either because the Higgs field is neutral.

Even if it cannot provide a physical interpretation for the Higgs field, does Miller's analogy nevertheless supply the basis for a physical interpretation of the Higgs boson? The phonon is the analogue of the Higgs boson. A phonon is a collective excitation or collective oscillation of the ions in the lattice. Consequently, the attempt to export this physical interpretation to the Higgs model encounters the same difficulty: if the Higgs boson is the collective effect of some collection of particles, these particles would have to play some role in the Standard Model. The Higgs boson is typically taken to be an elementary constituent of the Standard Model. In contrast, phonons are quasi-particles - they exhibit particle-like behaviour, but are not fundamental particles. ${ }^{26}$

There is another obstacle to transferring the physical interpretation of the phonon to the Higgs boson. The phonon is interpreted as being entirely an artifact of the medium-a clustering of the medium, as Miller explains. The conductor, BCS, and GL models employ non-relativistic quantum mechanics; the Higgs model uses relativistic QFT. Anderson recognized the implications for superconductivity models way back in his 1963 paper. In the superconductivity model considered by Anderson, the quasi-particles are plasmons. The solid state ground states are not relativistically invariant. A consequence is that there are different frequencies associated with the transverse and longitudinal components of the current in the superconductor (i.e., magnetic and electric components of polarizability are different), while relativistic invariance (Anderson presumes) prohibits this asymmetry in particle physics. A consequence of the different transverse and longitudinal frequencies in superconductors is that the transverse frequencies are ascribed to a heavy photon while the longitudinal frequencies are ascribed to a massive plasmon - and considered "entirely an attribute of the plasma" - though in both cases the mass arises from the interaction between the photon and the plasma. In Anderson's words, the transverse modes "are considered to result from modification of the propagation of real photons by the medium" (1963, p. 440). In a non-relativistic model, it is possible to interpret the transverse and longitudinal components differently, which is necessary to regard plasmons or phonons as being an ar-

\footnotetext{
${ }^{26}$ There is a substantial literature on why QFTs cannot be interpreted as describing particle-like entities. Ironically, one of the reasons for rejecting this interpretation is that quanta are not localizable to a finite region of spacetime, which is a property that is shared by quasi-particles of this type! From Anderson (1963): "In this case [i.e., conducting plasma type of vacuum], no net true charge remains localized in the region of the dressed particle; all of the charge is carried 'at infinity' corresponding to the fact, well known in the theory of metals, that all the charge carried by a quasi-particle in a plasma is actually on the surface. Nonetheless, conservation of particles, if not of bare charge, is strictly maintained" (p. 441).
} 
tifact of the medium while maintaining that the photons or electrons gain effective mass. In the historical context of the development of the Higgs model, these physical disanalogies between the non-relativistic superconductor models and the relativistic particle physics models was significant to particle physicists. As Higgs recalls many years later (commenting on Anderson (1963)): "However, he did not show that there was a flaw in the Goldstone theorem and he did not discuss any relativistic model, so particle theorists such as myself received his remark with skepticism" (2014, p. 851). Additional formal analogies between the non-relativistic superconductor models and the relativistic particle physics models had to be identified before particle physicists could trust the analogies to superconductivity.

Miller's analogy thus does not supply a physical interpretation for the Higgs field or Higgs boson. The physical disanalogies between the superconductivity models and the Higgs model generate the expectation that the formal analogies between the GL or BCS models and the Higgs model will not support the exportation of the physical interpretation to particle physics either. Does either the GL or BCS model defy these expectations? The closest formal analogue of the Higgs field is the condensate wave function $\psi(\mathbf{x})$ in the GL model, which represents the collection of superconducting electrons. $(\psi(\mathbf{x})$ is actually the analogue of the complex scalar field $\phi(x)$ that appears in the initial Lagrangian, prior to the application of the choice of gauge. The Higgs field $\rho(x)$ in the final Lagrangian is related to $\phi(x)$ by a gauge transformation and constant shift $v$. This point will be glossed over now, but deserves further scrutiny.) Since $\psi(\mathbf{x})$ is a quantum wave function in non-relativistic quantum mechanics and the Higgs field $\rho(x)$ is a quantum field, one would not expect the physical interpretation of $\psi(\mathbf{x})$ to carry over literally to $\rho(x)$. And, indeed, it does not seem to make sense to interpret $\rho(x)$ as representing a collection of particles (by analogy to the collection of superconducting electrons) for the same reason that it does not make sense to interpret the Higgs field as a collection of lattice ions in Miller's analogy.

The historically recognized set of formal analogies between the BCS and Higgs models does not identify an analogue of either the Higgs field or the Higgs boson (though the VEV of the Higgs field - the order parameter - does have an analogue). Very recently, it was recognized that the analogue of the Higgs boson in the BCS model is the AndersonBogoliubov collective mode. The Anderson-Bogoliubov mode is picked out as the analogue of the Higgs boson because "in some sense" it is the amplitude mode for the order parameter (Anderson, 2015, p. 93). That is, the (vertical) mathematical relationship between the order parameter $\Delta$ (the energy gap) and the Anderson-Bogoliubov mode in the BCS model maps on to the mathematical relationship between the order parameter $\langle 0|\phi(x)| 0\rangle$ and the Higgs boson in the Higgs model. Perhaps surprisingly, it took even longer to obtain experimental verification of the Anderson-Bogoliubov mode corresponding to the Higgs boson in a superconductor than it did to obtain experimental evidence for the Higgs boson! (See Anderson (2015) and Sherman et al. (2015).) Does the Anderson-Bogoliubov collective mode supply an analogical template for physically interpreting the Higgs boson? As Anderson explains, the Anderson-Bogoliubov mode "is not an actual particle" due to its collective origin; it is a collective property of the superconductor. Thus, as in Miller's analogy, the collective nature of the Anderson-Bogoliubov mode is at odds with the elementary nature of 
the Higgs boson: the Higgs boson is itself an elementary constituent, not a collection of other elementary constituents, so is not given the same interpretation as the Anderson-Bogoliubov mode.

There is a final twist to this formal analogy between the Higgs boson and the AndersonBogoliubov mode. As already noted, there is no formal analogue of the Higgs field in the BCS model. Reflecting on the formal analogy between the Anderson-Bogoliubov mode and the Higgs boson, Anderson speculates (in 2015)

If superconductivity does not require an explicit Higgs in the Hamiltonian to observe a Higgs mode, might the same be true for the $126 \mathrm{GeV}$ mode [attributed to the Higgs boson]? As far as I can interpret what is being said about the numbers, I think that is entirely plausible. Maybe the Higgs boson is fictitious! (2015, p. 93)

That is, there is no analogue of the Higgs field in the Hamiltonian (or Lagrangian) for the BCS model. Anderson suggests that if it were possible - by formal analogy with the BCS model - to write the Lagrangian for the EW theory in Higgs-less form, then by the standards of physical interpretation that prevail in particle physics, there would be no Higgs boson! Instead of transferring the physical interpretation from the BCS model to the Higgs model, Anderson restricts his argument to the formal analogy and applies the standards of physical interpretation established in particle physics. In quantum field theories, the mass of an elementary particle appears in the coefficient of the squared term of the field in the Lagrangian. For example, recall that the Lagrangian (13) in the textbook presentation of the Higgs mechanism was interpreted as having a mass of $g v$ because the Lagrangian contains the term $\frac{1}{2}(g v)^{2} B_{\mu} B^{\mu}$. However, there is no Higgs term in the BCS Hamiltonian; thus, if there turns out to be a formally analogous Lagrangian for the Higgs model, then there would turn out to be no Higgs boson! $!^{27}$ If this line of formal analogical reasoning were to pan out, it would be a fitting finale to the saga of formal analogies to models of superconductivity informing the development of the Higgs model.

\subsection{Conclusion}

We have argued that the analogy between SSB in superconductivity and the Higgs mechanism is strictly formal - there is no material or physical analogy. Due to the lack of temporal structure in the Higgs mechanism, the modality of SSB is different in the two cases, leading to the absence of causal processes in the Higgs mechanism. This definitively rules out a material analogy with superconductivity. Physical analogies are undermined in several ways: the atemporality of the Higgs mechanism, the failure of the Higgs mechanism to fit on either side of the dynamical-phenomenological distinction between the BCS and GL models, and the failure of elementary gauge bosons to 'gain' mass in some sort of causal process. The

\footnotetext{
${ }^{27}$ N.B. This theory would still predict the experimental results that were taken to confirm the Higgs boson - as the BCS model (or a refined successor of it) predicts the experimentally-measured AndersonBogoliubov mode.
} 
formal analogy to the Higgs mechanism - as it currently stands in the Standard Model - is tighter with the GL model than the BCS model; it may perhaps be fruitful to pursue a successor theory in which the formal analogy is a better fit with the BCS model. These are known as models of dynamical symmetry breaking, to which we turn our attention in the next section.

\section{Pursuing a closer analogy with the BCS model: 'Dy- namical' models of EW SSB}

The interest in developing an alternative to the Higgs model that is a closer analogue to the BCS theory was natural from a heuristic standpoint: major progress had been made by exploiting an analogy between BCS theory and the Dirac equation, so why not push the analogy further? With the advent of SSB in particle physics, the floodgates of analogy seemed to open. Some physicists regard SSB as the most important advance in the development of the Standard Model. In the words of Jona-Lasinio: "Although [SSB] pervaded the physics of condensed matter for a very long time, magnetism is a prominent example, its formalization and the recognition of its importance has been an achievement of the second half of the XXth century" (Jona-Lasinio, 2010, p. 2). The close formal analogy between the phenomenological GL model and the Higgs mechanism, accompanied by the dynamical account of SSB in the BCS model, inspired many particle physicists to pursue so-called dynamical symmetry breaking models in particle physics (e.g., technicolor models). This particular strategy for model-building was also motivated by other perceived shortcomings in the Higgs model. For example, Lane lists the following motivations for technicolor:

1. Elementary Higgs models provide no dynamical explanation for electroweak symmetry breaking.

2. Elementary Higgs models are unnatural, requiring fine tuning of parameters to enormous precision.

3. Elementary Higgs models with grand unification have a hierarchy problem of widely different energy scales.

4. Elementary Higgs models are trivial.

5. Elementary Higgs models provide no insight to flavor physics.(Lane, 2002, p. 3)

Our analysis in Sec. 5.4 entails that - in spite of the name - models of EW SSB that are based on a closer analogy with the BCS model are not dynamical models in the usual sense of the term. The BCS model is appropriately labeled "dynamical" because it supplies a causal-dynamical account of the process of SSB described by the phenomenological GL model. The Higgs model cannot be classified as a phenomenological model because (among other reasons) it does not describe a temporal process of SSB; in contrast to the GL model, the Higgs model does not demand a causal-dynamical model to underpin it (pace Lane's 
first motivation). However, we shall stick to the conventional nomenclature of 'dynamical' SSB models in order to avoid confusion. The models examined in this section attempt to go beyond the Higgs component of the Standard Model by positing a composite particle to replace the elementary Higgs boson, in analogy to the Cooper pair bosons in the BCS model. The goal is to develop an empirically adequate 'dynamical' model that is only dynamical insofar as it aspires to the second and third characteristics of the dynamical BCS model: a focus on micro-constituents (as opposed to collective phenomena) and theoretical inputs (as opposed to empirical inputs).

There is an additional reason that 'dynamical' SSB models should not be regarded as underpinning the Higgs model. The set of historical analogies between the BCS model and the Higgs model does not include the Higgs boson (see Table 3). 'Dynamical' models of SSB draw an analogy between the Higgs boson and the Cooper pair boson. This analogy is not compatible with the set of historical analogies between the BCS model and the Higgs model. Thus, 'dynamical' models do not extend the Higgs model by consistently extending the set of analogies with the BCS model. Note that this apparently undermines the first of Lane's motivations above: though it is true that the Higgs mechanism does not provide a dynamical explanation for SSB, neither does it leave theorists wanting a dynamical underpinning.

There are two possible categories into which any empirically successful successor to the Higgs model might fall:

1. A successor model in which there is a genuine temporal and causal process of EW SSB. The Lagrangian will take a different form from the Higgs Lagrangian. The model approximately matches correct predictions made by the Standard Model at low energies, and will generate better or more accurate predictions where the Standard Model breaks down at higher energies. In this case, the correct interpretation would be that this new model is not a dynamical account of the Higgs mechanism as it stands in the Standard Model. This would be a new, replacement model, in which SSB occurs as a consequence of some dynamical process. The model could also supply a causal-dynamical explanation for the occurrence of SSB.

2. A successor model in which there is no temporal (or causal) process of EW SSB. A model of this type could still provide new insight into physics in the EW sector (e.g. that the Higgs boson is actually a composite particle, or that the EW Lagrangian does not include the Higgs field).

The 'dynamical' SSB models examined in this section are (in some cases empirically unsuccessful) examples of category 2 models: the Higgs boson as a quark composite, electroweak perturbation, and technicolor. These models were guided by analogies to Cooper pairs in the BCS model. Category 1 models could include other models that radically alter the physics of the Standard Model (e.g., string theory, loop quantum gravity, finite temperature field theory, etc.). In particular, finite temperature field theory does aim to incorporate a temperature-dependent process of SSB. However, as far as we are aware, none of these approaches to model-building beyond the Standard Model are premised on exploiting an 
analogy with Cooper pairs in the BCS model. We stress that our arguments in this section are not intended to apply to finite temperature field theory or other attempts to develop new physics that includes genuinely dynamical processes of SSB; we restrict our attention to category 2 models motivated by analogies to the BCS model.

The success of any analogy in science - whether formal, physical, or material - must be judged on the basis of the empirical adequacy of the model it generates. The first two 'dynamical' models in this section have already been rejected due to empirical inadequacytheir predictions did not match experimental findings - while current minimal technicolor models have yet to have those predictions which diverge from the Standard Model testedthough a certain class of models with (relatively) low-energy divergences from the Standard Model have been ruled out by recent experiments at the LHC (Stöltzner, 2014).

\subsection{The Higgs boson as a quark composite}

In Marshak's introductory particle physics book, the introduction to SSB is given via an Abelian $(U(1))$ Higgs mechanism as in Sec. 3.3 above (Marshak 1993, Sec. 4.4a). After introducing the basic mechanism - in which the choice of a particular gauge is exploited to make explicit the manifestation of mass in the otherwise massless Nambu-Goldstone boson - Marshak describes the application of "a quasi-Higgs SSB mechanism operating within the framework of the $U(1)$ gauge theory of electromagnetism"-low temperature superconductivity. Quasi-Higgs here means that, unlike the Higgs model, this model has a composite boson in analogy to the composite Cooper pairs in the BCS model.

As Marshak explains, "From the vantage point of particle physics, the inverse of $[l$ - the coherence length] can be identified with the 'quasi-Higgs mass' of the 'Cooper pair'... the inverse of $d$ [the penetration depth] is clearly of the form $g \nu$ and has been identified with the (transverse) magnetic modes of the 'heavy photon'" (Marshak, 1993, p. 223). Thus

the equations describing superconductivity can be presented in such a way as to evoke the analogy to QFT. The analogy is pushed too far - from formal to material - when Marshak insinuates that it is the same mechanism behind phenomena in condensed matter and particle physics. They share the same mathematical structure, but we cannot conclude from this that the physical content is the same. Though the composite Higgs model shares greater formal similarity with the BCS model, this is no guarantee of a physical analogy.

A key physical difference between superconductivity and the Higgs mechanism is that, in electroweak symmetry breaking, the boson that emerges (the Higgs boson) is typically taken to be an elementary particle, while the superconducting boson (the Cooper pair) is a fermion condensate. At Marshak's time of writing, however, this was not well established, and this model postulates that the Higgs boson is actually a top quark condensate. Marshak reasons that, since the acquisition of mass by the Nambu-Goldstone bosons "due to the Higgs mechanism can be activated by a 'fermion condensate' (serving as a 'quasi-Higgs particle') or result directly from the intervention of an 'elementary' Higgs particle," (p. 219) and "the mass of the still-unobserved t quark is moving upward to the range of $100 \mathrm{GeV}(>92$ $\mathrm{GeV}$ ) [towards half the suspected Higgs scale], the Higgs mechanism for the SSB of the electroweak group may, after all, be modelled after low temperature superconductivity" 
(Marshak, 1993, p. 226). This model is now obsolete since we now know that the top quark mass $\left(m_{t}=173.3 \mathrm{GeV}\right)$ exceeds that of the Higgs. Thus the possibility of the Higgs being a top quark condensate has been ruled out.

\subsection{Electroweak perturbation}

Quigg, in his paper on the progress of SSB in physics (Quigg, 2007) also describes some approaches to dynamical electroweak symmetry breaking, which draw on an analogy with BCS theory and branch off from Nambu's effective theory of QCD. Since Nambu's effective theory is more closely analogous to the BCS model than the resulting EW theory, this too counts as an attempt to form a tighter analogy with the BCS model. The first approach described is a direct attempt to mimic BCS theory, which "interprets the dynamical origin of the order parameter through the formation of correlated states of elementary fermions, the Cooper pairs of electrons" (Quigg, 2007, p. 1031). This approach treats the electroweak interaction, $S U(2)_{L} \times U(1)_{Y}$, as a perturbation to the strong force, since the strong force has much greater magnitude. As Quigg explains, "When we turn on the $S U(2)_{L} \times U(1)_{Y}$ electroweak interaction, the electroweak gauge bosons couple to the axial currents and acquire masses of order $\sim g f_{\pi}$ ", where $g$ is the weak coupling constant, and $f_{\pi}$ is the pion decay constant, which is taken to be the coupling strength of the interaction between pions and and the broken generators (Quigg, 2007, p. 1031). The mass ratios obtained from this Nambu-like approach are in close agreement with those predicted by the conventional GWS theory, with $M_{\gamma}=0$, and

$$
\frac{M_{Z}^{2}}{M_{W}^{2}}=\frac{1}{\cos ^{2} \theta_{W}}
$$

Though this theory gets the qualitative features right, "[t]he masses acquired by the intermediate $[W$ and $Z]$ bosons are 2500 times smaller than required for a successful lowenergy phenomenology; the $W$-boson mass is only $M_{W} \approx 30 \mathrm{MeV}$, because its scale is set by $f_{\pi}$ " (Quigg, 2007, p. 1032). Thus, this straightforward dynamical model is also ruled out by empirical results.

\subsection{Minimal Technicolor}

Both of the approaches described above were attempts to extend the formal analogy with the BCS model, and both failed to do so; they simply do not match up to known empirical data. In the case of the quark composite model, the Higgs mass is not in the vicinity to be consistent with quark condensates, while the electroweak perturbation model grossly underestimates the mass of the $W$ and $Z$ bosons. The simplest approaches are thus abandoned, but the BCS idea of dynamical symmetry breaking remains in a minimal technicolor model, which would include not only the electroweak sector but QCD as well. In this theory a new interaction - technicolor - is introduced, and the breaking of chiral technicolor symmetry leads to a triplet of Goldstone bosons analogous to the pion triplet in Nambu's effective theory of QCD. Since these 'technipions' have not been observed, there is a freedom in choosing their decay constant, and it is chosen such that the Standard Model masses of the 
weak vector bosons, formed from the technipions when the electroweak interaction is 'turned on', are recovered. It is worth noting that there also exist a large number of technicolor descendents - theories whose origin stems from variations to a minimal technicolor theory. Stöltzner (2014), in conjunction with the Wuppertal research group "Epistemology of the LHC," categorizes these models as dynamical symmetry breaking models of mass generation. They share a guiding principle motivated by a 'dynamical' account of SSB, but differ widely in their execution. There is a wide range of models in this category that posit additional particles and forces which have been neither confirmed nor disconfirmed by experiment.

Proponents of the minimal technicolor model argue that it has theoretical virtues. For example, Lane (quoted on p.36) argues that the model has greater explanatory power and simplicity. Others have cast doubt on some of these claims. For example, Quigg points out that even if the explanation of the origin of vector boson mass is achieved, the model "offers no explanation for the origin of quark and lepton masses, because no Yukawa couplings are generated between Higgs fields and quarks of leptons" (Quigg, 2007, p. 1051), and leaves us with a further case of unexplained SSB. In any case, as with any application of analogical reasoning as a heuristic, the ultimate determinant of whether the minimal technicolor model (or one of its descendants) will be accepted is whether its novel empirical prediction of new particles and forces are borne out by experiment.

Suppose that, at some point in the future, experimental results provide confirmation for the minimal technicolor model. On the positive side, there will be a physical analogy between the Cooper pairs in the BCS model and the Higgs boson in the minimal technicolor model; the physical similarity is that both Cooper pairs and the Higgs boson are composed of more fundamental particles. This explains the origin of mass of the Higgs boson as a composite of elementary components. However, for all of the reasons laid out already, the minimal technicolor model does not supply an account of a causal process of SSB in the course of which the Higgs boson acquires mass. The explanation of the compositionality of various particles (Higgs boson, $W^{ \pm}$and $Z$ bosons) and the description of a new force of nature would be substantial accomplishments, but would not supply a causal or dynamical explanation of mass acquisition. We urge that this situation should in no way be regarded as problematic. Once it is recognized that the dynamical-phenomenological distinction that holds between the BCS and GL models does not carry over to particle physics, the Higgs model is not merely phenomenological, and so does not require a dynamical model to underpin it. Indeed, we would go even further and draw the moral that the heuristic strategy of using analogies to develop new models need not be based on physical analogies; purely formal analogies can play this role in some cases. The Higgs model - which has arguably been successful, even it eventually is succeeded by another model - is a case in point.

\section{Conclusion}

The historical record provides ample evidence that analogies between superconductivity and particle physics played an important heuristic role in the development of the Higgs model. We have distinguished analogies to the GL and the BCS models of superconductivity, re- 
spectively, and have attempted to carefully disentangle the purely formal analogies from the potential material and physical analogies. Our general conclusion is that both the set of analogies between the GL and the Higgs models and the set of analogies between the BCS and the Higgs models are formal; neither is physical nor material. Consequently, neither analogies to the GL model nor analogies to the BCS model furnish a basis for a physical interpretation of the Higgs model.

Material analogies to the GL model are ruled out at the outset because the GL model does not describe causal structure. The purely formal nature of the analogies to the GL model was indicated by the mathematical formalism on which the analogies were based: the form of the potential in the Higgs Lagrangian and the GL expression for free energy. The reason that these expressions take the same form is that both result from minimal expansions in which higher order terms are discarded, which suggests that the similarity is not due to the fact that the expressions represent the same physical content. A stronger reason for characterizing the analogies as purely formal - and not physical - is that there are a number of physical disanalogies between the formal analogues. The formal analogy does not map temporal processes to temporal processes; space in the GL model is mapped to spacetime in the Higgs model. The effective mass gain of photons and quasi-particles is a dynamical process in the GL model, while $W$ and $Z$ bosons and the Higgs boson do not gain mass in a dynamical process in the Higgs model. These physical disanalogies undermine a potential physical analogy. The physical interpretation of the GL model cannot be exported to the Higgs model. $\psi(\mathbf{x})$, the condensate wave function in the GL model, is the formal analogue of the Higgs field. $\psi(\mathbf{x})$ represents a collection of particles; the Higgs field cannot similarly be interpreted as representing a collection of particles.

The analogies between the BCS and Higgs models are also purely formal. The BCS model offers a causal explanation of SSB; however, there is no material analogy between the description of the causal process of SSB in superconductors and SSB in the Higgs model. This is because SSB in the Higgs model is not a temporal process and a fortiori not a causal process. A further consideration is that the Cooper pairs, which are the linchpin of the BCS causal explanation of SSB, have no analogue in the Higgs model. Physical disanalogies again supply evidence that the analogies between the BCS and Higgs models are not physical analogies, but purely formal analogies. Recently it was realized that the formal analogue of the Higgs boson is the Anderson-Bogoliubov mode. However, the AndersonBogoliubov mode does not supply a template for physically interpreting the Higgs boson, which is generally understood to be an elementary particle and not a collective phenomenon. Anderson speculates that - if the formal analogy with the BCS model is pursued to its ultimate conclusion - it will turn out that the Higgs boson is fictitious in the sense that the Higgs field with which it is associated will not appear in the Lagrangian.

We also examined three 'dynamical' models of EW SSB which have been proposed as successors to the Standard Model and which are based on analogies to the BCS model that are tighter than those between the BCS and Higgs models. One of the original motivations for developing 'dynamical' models of SSB was to find a dynamical model underlying the Higgs model. Because the dynamical-phenomenological distinction that holds between the 
GL and BCS models does not carry over to particle physics, 'dynamical' models of SSB do not supply a dynamical underpinning for the Higgs model in the manner that the BCS model supplies a dynamical underpinning for the GL model. Furthermore, the analogies to the BCS model invoked by the 'dynamical' models of SSB are inconsistent with the historical analogies to the BCS model that informed the Higgs model. 'Dynamical' models do represent deeper structure, however, in the sense that the bosons are replaced by composite particles. The minimal technicolor model (and its descendants) have yet to be either confirmed or disconfirmed by empirical evidence. If the minimal technicolor model is ultimately vindicated by experiment, then there would be physical analogies between the Cooper pairs in the BCS model and the pion triplets, but these physical analogies would be accompanied by the aforementioned material disanalogies. (The fact that SSB is not a causal-dynamical process in minimal technicolor models does not, of course, entail that it is impossible to devise a model in which EW SSB is a causal-dynamical process. This is the goal of finite temperature field theory, for example.)

Returning to the Standard Model, our conclusion is that formal analogies - rather than material or physical analogies - to superconductors guided the construction of the Higgs model. This conclusion might raise the worry (especially for scientific realists) that the success of analogical reasoning in this case is left a mystery. Unquestionably, the Higgs model has proven successful (even it gets supplanted by a successor model sometime in the future) and analogies to superconductivity played a crucial role in the development of the Higgs model. What is the explanation for the similarities in the formal structures used in superconductor and EW models, if not physical or causal similarities between the systems represented by these formal structures? Those whose distinctions we borrowed to frame this case study, namely Hesse (1966) and Bartha (2010), would share this worry. However, a closer examination of the historical situation reveals why formal analogies were needed and why physical and material analogies would have been inappropriate. The problem in particle physics circa 1960 was that there were a number of misconceptions about the representative capacity of gauge theories with spontaneously broken symmetry: for example, that gauge bosons are massless and that SSB in a gauge theory is necessarily accompanied by massless gauge bosons. Formally, the BCS model is a gauge theory with spontaneously broken symmetry. Physicists first contemplated the BCS model of superconductivity as an analogue for particle physics models because the gauge bosons are effectively massive. This suggested the possibility of models of SSB in particle physics with massive gauge bosons. This possibility was realized through the application of the formal analogies discussed in this paper. Physical and material analogies to superconductivity would not have been appropriate for addressing these misconceptions about the formalism because the mass acquisition that was at the heart of the misconceptions in particle physics was not a physical process. The mass gain that physicists examined was mass gain relative to counterfactual theories. The formal analogy drew attention to a new range of possibilities for applying the formalism, re-opening avenues that had been mistakenly foreclosed. In fact, a stronger point could arguably be made: the physical disanalogies between superconductivity and particle physics actually contributed to the heuristic usefulness of the formal analogy because the key features of the 
superconductivity model were accessible to experiment as a result of physical disanalogies. For example, the Meissner effect: beyond a small penetration depth, neither electric nor magnetic fields will be present within the superconductor, and the short range of the electromagnetic interaction is regarded as an indication of the effective mass of a photon. From the vantage point of the completed Higgs model, the initial suspicion that there could be a physical analogy between the effective mass of gauge bosons in superconductivity and the mass of gauge bosons proved baseless; there is only a superficial physical similarity between effective mass in superconductors and mass in EW interactions. However, the morals about the representative capacity of the formalism in particle physics remained valid.

Another reason that merely formal analogies were heuristically useful in this case is that the formal tools on which the formal analogies were based-perturbative expansion of expressions for energy around a ground state, gauge theories, symmetries, SSB - have a wide scope of applicability across different physical domains. These formal tools are general and abstract; physical content particular to a domain is introduced by, for instance, choosing a particular form for the Lagrangian or considering particular symmetries, and, moreover, by giving a domain-specific physical interpretation of the formalism. Contrast this explanation of the success of gauge theories with spontaneously broken symmetry with that offered by Marshak:

While it is prudent not to push analogies between condensed matter and particle physics too far, the fact remains that the Ginzburg-Landau equation is equivalent to the Lagrangian of an Abelian gauged charged scalar field and that both undergo SSB. The perceived relationship is certainly a tribute to our present-day understanding of physical phenomena since the same SSB Higgs (or quasi-Higgs) mechanism are invoked in condensed matter and particle physics despite the vast disparity in energy scale [fractions of eVs in superconductivity in contrast to hundreds of GeVs in particle physics - a factor of $10^{12}$ or more]. (p. 224)

We agree that it is not prudent to push analogies too far. We submit that this is a case in which our success across scales reflects our success in understanding the formalism and its structure, rather than an understanding of common physics of the physical phenomena.

A final reason that formal analogies to superconductivity were useful was because solid state physicists had a much clearer physical interpretation of the physics underlying superconductivity phenomenology than particle physicists did of that underlying particle phenomenology. Having an intuitive picture that goes along with a mathematical formalism can aid with manipulating the mathematics. An abstract mathematical formalism can be compatible with more than one intuitive physical picture (e.g., the wave equation). The intuitive picture of the physical processes in superconductivity is inapplicable to particle physics, but was still helpful in enabling physicists to grasp the range of consequences of the formalism (e.g., massive gauge bosons). As long as the physical intuitions from the source domain are not read literally as supplying a physical interpretation for the formalisms in the target domain, they can be helpful for manipulating the same formalism in the new domain.

What, then, are we to make of appeals to analogies to superconductors to explain the Higgs mechanism to the public or to students? The analogies do not illuminate the physical 
interpretation of the Higgs boson or the Higgs field and do not furnish a mechanism for mass acquisition. For physics students, textbook presentations of the analogies can serve the same purpose as for professional physicists: the intuitive physical picture of spontaneous symmetry breaking in superconductors gives students something to visualize when they are applying a similar formalism in the Higgs model. What does the public (including politicians) gain from this type of analogy? Miller's analogy to conductors does yield a type of explanation, but not an ontological explanation. He offered the analogy as a response to William Waldegrave's question "What is the Higgs boson, and why do we want to find it?" The analogy does address the second part of the question: as for any set of analogies-material, formal, or physical-used as a heuristic to develop a new theory, the set of analogies described by Miller raise the Higgs model as a theoretical possibility, but do not provide evidence that this possibility is actualized. Empirical evidence is needed to confirm that, among the possible models, the Higgs model is the correct one. Miller's analogy illustrated the missing piece of the puzzle, and supplied the requested explanation for building the LHC. 


\section{References}

P. Anderson. Plasmons, gauge invariance, and mass. Physical Review, 130:439-442, 1963.

P. Anderson. Concepts in solids: lectures on the theory of solids, volume 58. World Scientific, 1997.

P. Anderson. Higgs, Anderson and all that. Nature Physics, 11:93, 2015.

J. Bardeen, L. Cooper, and J. R. Schrieffer. Theory of superconductivity. Physical Review, 108(5):1175, 1957.

P. Bartha. By Parallel Reasoning: The construction and evaluation of analogical arguments. Oxford University Press, 2010.

L. M. Brown and T. Y. Cao. Spontaneous breakdown of symmetry: Its rediscovery and integration into quantum field theory. Historical Studies in the Physical and Biological Sciences, 21(2):211-235, 1991.

T. Y. Cao, editor. Conceptual Foundations of Quantum Field Theory. Cambridge University Press, 1999.

E. Castellani. On the meaning of symmetry breaking. In K. Brading and E. Castellani, editors, Symmetries in Physics. Cambridge University Press, 2003.

S. Coleman. Aspects of Symmetty: Selected Erice Lectures. Cambridge University Press, 1985.

J. Earman. Laws, symmetry and symmetry breaking: Invariance, conservation principles and objectivity. Philosophy of Science, 71(5):1227-1241, 2002.

J. Earman. Rough guide to spontaneous symmetry breaking. In K. Brading and E. Castellani, editors, Symmetries in Physics. Cambridge University Press, 2003.

J. Earman. Curie's principle and spontaneous symmetry breaking. International Studies in the Philosophy of Science, 18:173-198, 2004.

G. Emch. Quantum statistical physics. In J. Butterfield and J. Earman, editors, Philosophy of Physics, Part B. Elsevier, 2007.

Y. Gingras. The creative power of formal analogies in physics: the case of Albert Einstein. Science and Education, 24:529-541, 2015.

V. Ginzburg and L. Landau. On the theory of superconductivity. Springer, 2009.

J. Goldstone. Field theories with superconductor solutions. Il Nuovo Cimento, 19(1):154$164,1961$. 
J. Goldstone, A. Salam, and S. Weinberg. Broken symmetries. Physical Review, 127(3):965, 1962.

L. Gor'kov. Microscopic derivation of the Ginzburg-Landau equations in the theory of superconductivity. Journal of Experimental and Theoretical Physics, 36:1364-1367, 1959.

R. Haag. The mathematical structure of the Bardeen-Cooper-Schrieffer model. Il Nuovo Cimento, pages 287-299, 1962.

M. B. Hesse. Models and Analogies in Science. University of Notre Dame Press, 1966.

P. Higgs. Broken symmetries, massless particles and gauge fields. Physics Letters, 12(2): 132-133, 1964.

P. Higgs. Spontaneous symmetry breakdown without massless bosons. Physical Review, 145 (4):1156-1163, 1966.

P. Higgs. Nobel lecture: Evading the Goldstone theorem. Reviews of Modern Physics, 86: 851-853, 2014.

L. Hoddeson, editor. The Rise of the Standard Model: A History of Particle Physics from 1964 to 1979. Cambridge University Press, 1997.

K. J. Holyoak and P. Thagard. Mental Leaps: Analogy in Creative Thought. MIT Press, 1995.

G. Jona-Lasinio. Cross fertilization in theoretical physics: the case of condensed matter and particle physics. In K. Brading and E. Castellani, editors, Symmetries in Physics. Cambridge University Press, 2003.

G. Jona-Lasinio. Analogies in theoretical physics. arXiv:1003.4170v1, 2010.

L. Kadanoff. Slippery wave functions. Journal of Statistical Physics, 152(5):805-823, 2013.

K. Koraca. Practical unification of solid-state and particle physics. In EPSA11 perspectives and foundational problems in philosophy of science, pages 253-264, 2011.

P. Kosso. The epistemology of spontaneously broken symmetries. Synthese, 122:359-376, 2000.

K. Lane. Two lectures on technicolor. In l'Ecole de GIF at LAPP, Annecy-le-Vieux, France, 2002.

M. Le Bellac. Thermal Field Theory. Cambridge University Press, 2000.

H. Lyre. Does the Higgs mechanism exist? International Studies in the Philosophy of Science, 22(2):119-133, 2008. 
R. Marshak. Conceptual Foundations of Modern Particle Physics. World Scientific, 1st edition, 1993.

C. Martin. On continuous symmetries and the foundations of modern physics. In Brading and Castellani, editors, Symmetries in Physics. 2003.

D. Miller. Politics, solid state and the higgs. Physics World, September 1993.

M. Morrison. Spontaneous symmetry breaking: Theoretical arguments and philosophical problems. In K. Brading and E. Castellani, editors, Symmetries in Physics. Cambridge University Press, 2003.

M. Morrison. Emergent physics and micro-ontology. Philosophy of Science, 79(1):141-166, 2012.

Y. Nambu. Spontaneous symmetry breaking in particle physics: A case of cross fertilization. Nobel Lecture, 2008.

C. Quigg. Spontaneous symmetry breaking as a basis of particle mass. Reports on Progress in Physics, 70(7):1019, 2007.

L. Ruetsche. Interpreting Quantum Theories. Oxford University Press, 2011.

D. Sherman, U. S. Pracht, B. Gorshunov, S. Poran, J. Jesudasan, M. Chand, P. Raychaudhuri, M. Swanson, N. Trivedi, A. Auerbach, M. Scheffler, A. Frydman, and M. Dressel. The Higgs mode in disorded superconductors close to a quantum phase transition. Nature Physics, 11:188-192, 2015.

C. Smeenk. The elusive Higgs mechanism. Philosophy of Science, 73(5):487-499, 2006.

M. Stöltzner. Higgs models and other stories about mass generation. Journal for General Philosophy of Science, 45:369-386, 2014.

W. Struyve. Gauge invariant accounts of the Higgs mechanism. Studies in History and Philosophy of Science Part B: Studies in History and Philosophy of Modern Physics, 42 (4):226-236, 2011.

J. O. Weatherall. Understanding gauge. Forthcoming, http://philsci-archive.pitt.edu/11449/, 2015.

S. Weinberg. A model of leptons. Physical Review Letters, 19:1264, 1967.

S. Weinberg. Superconductivity for particular theorists. Progress of Theoretical Physics Supplement, 86:43-53, 1986.

E. Witten. From superconductors and four-manifolds to weak interactions. Bulletin of the American Mathematical Society, 44(3):361-391, 2007. 
A. Wuthrich. Eating Goldstone bosons in a phase transition: a critical review of Lyre's analysis of the Higgs mechanism. Journal for General Philosophy of Science, 43:281-287, 2012. 